\title{
THE DERIVATIVES OF HOMOTOPY THEORY
}

\author{
BRENDA JOHNSON
}

\begin{abstract}
We construct a functor of spaces, $M_{n}$, and show that its multilinearization is equivalent to the $n$th layer of the Taylor tower of the identity functor of spaces. This allows us to identify the derivatives of the identity functor and determine their homotopy type.
\end{abstract}

The calculus of homotopy functors, developed by Goodwillie ([G1], [G2], [G3]), establishes that a homotopy functor, $F$, satisfying certain connectivity conditions, has associated to it a tower of functors, $\ldots \rightarrow P_{n} F \rightarrow P_{n-1} F \rightarrow \ldots$. These functors act like a Taylor series approximation to $F$ in the sense that for a space, $X$, there is a map, $p_{n} F(X): F(X) \rightarrow P_{n} F(X)$, for each $n$, and the connectivity of this map increases with $n$. This theory has been applied to the study of the functor $A$, Waldhausen's algebraic $K$-theory of spaces.

In this paper, we turn our attention to the Taylor tower of the identity functor of spaces, $I$. The ultimate goal is to identify the Taylor tower of $I$ and use it to study homotopy theory. In this paper, we construct a collection of symmetric functors, $\left\{M_{n}\right\}$, and show that the multilinearization of $M_{n}$ is equivalent to the $n$th layer, fiber $\left(P_{n} I \rightarrow P_{n-1} I\right)$, of the Taylor tower of $I$. This construction also allows us to identify the $n$th derivative of $I$. This is a spectrum with $\Sigma_{n}$ action which is equivalent to the functor fiber $\left(P_{n} I \rightarrow P_{n-1} I\right)$.

The paper is organized as follows. In section 1 we summarize the basic results and terminology of calculus that will be used throughout the paper. In section 2 we describe the problem of finding the Taylor tower of $I$ in more detail and state the main results. In section 3 we outline the method by which the $n$th derivative of a functor is determined. In section 4 we construct the functor $M_{n}$ and a natural transformation, $T_{n}$, used to establish the equivalence between the multilinearization of $M_{n}$ and fiber $\left(P_{n} F \rightarrow P_{n-1} F\right)$. In section 5 we determine the homotopy type of the derivatives, and in section 6 we show that $T_{n}$ is sufficiertly connected to induce an equivalence between the multilinearization of $M_{n}$ and fiber $\left(P_{n} F \rightarrow P_{n-1} F\right)$.

The results in this paper come from the author's thesis, written under the direction of Tom Goodwillie at Brown University. The author wishes to thank him for his guidance and for many insightful discussions. The author also wishes to thank Randy McCarthy for his helpful suggestions during the writing of this paper.

Received by the editors February 10, 1994.

1991 Mathematics Subject Classification. Primary 55P65. 


\section{THE TAYLOR TOWER OF A FUNCTOR}

To start, we need to describe the context in which we will be working. We need both to establish some notation and terminology and to describe (in brief) the language and main results of Goodwillie's calculus of homotopy functors which will be used throughout this paper. We will not give a complete exposition of the theory of calculus of homotopy functors. Instead we will outline the terms and results needed in this work. For further detail, explanation and examples the reader is referred to Goodwillie's papers ([G1], [G2], and [G3]). Specifically, [G2] contains results about $n$-cubes of spaces, excision, and analytic functors, while [G3] contains results about the Taylor tower of a functor.

We start with the conventions. By a space (generally denoted $X$ ) we will mean an object in one of two categories. For the general results from Goodwillie's works, a space $X$ will be an object in the category of compactly generated topological spaces. For the specific results concerning the derivatives of the identity functor, a space will be a topological space having the homotopy type of a finite $\mathrm{CW}$-complex. Moreover, we will assume that all the spaces considered have non-degenerate basepoints. When we say that two spaces are equivalent, we will mean that they are weakly homotopy equivalent. By the suspension of a space, $\Sigma X$, we will mean the reduced suspension, $S^{1} \wedge X$.

By an $n$-cube of spaces we will mean the following. Let $\underline{n}$ denote the set $\{1,2, \ldots, n\}$. Let $N$ be the category whose objects are the subsets of $\underline{n}$ and whose morphisms are the inclusion maps among the subsets. An $n$-cube of spaces is a covariant functor from the category $N$ to the category of spaces.

Goodwillie defines and uses particular $n$-cubes of spaces, namely Cartesian and co-Cartesian diagrams. Cartesian can be defined in several ways. One way is the following. Let $\mathbf{X}$ be an $n$-cube of spaces. Let

$$
h_{0}(\mathbf{X})=\operatorname{holim}_{K \subseteq \underline{n}, K \neq \varnothing}(\mathbf{X}(K)),
$$

where holim means the homotopy inverse limit (as in $[\mathrm{B}-\mathrm{K}])$. There is a map $a(\mathbf{X})$ defined as the composition $a(\mathbf{X}): \mathbf{X}(\varnothing)=\lim \mathbf{X} \stackrel{\simeq}{\longrightarrow}$ holim $\mathbf{X} \rightarrow h_{0}(\mathbf{X})$. If $a(\mathbf{X})$ is an equivalence then we say that $\mathbf{X}$ is Cartesian. If $a(\mathbf{X})$ is $k$-connected, we say that $\mathbf{X}$ is $k$-Cartesian.

Dually, we define co-Cartesian. Let $\mathbf{Y}$ be an $n$-cube. Let

$$
h_{1}(\mathbf{Y})=\underset{K \subset \underline{n}, K \neq\{1, \ldots, n\}}{\operatorname{hocolim}} \mathbf{Y}(K),
$$

where hocolim denotes the homotopy colimit (as in [B-K]). There is again a map $b(\mathbf{Y}): h_{1}(\mathbf{Y}) \rightarrow \mathbf{Y}(\{1, \ldots, n\})$. If $b(\mathbf{Y})$ is an equivalence, then we say that $\mathbf{Y}$ is a co-Cartesian $n$-cube. If $b(\mathbf{Y}): h_{1}(\mathbf{Y}) \rightarrow \mathbf{Y}(\{1, \ldots, n\})$ is $k$-connected then we say that $b(\mathbf{Y})$ is $k$-co-Cartesian. An $n$-cube is strongly co-Cartesian if each of its 2-faces is co-Cartesian.

Cartesianness and co-Cartesianness are related to a certain extent. A classical result, the Blakers-Massey theorem, estimates the degree to which a co-Cartesian square is Cartesian as a function of the connectivity of the maps $\mathbf{X}(\varnothing) \rightarrow \mathbf{X}(\{1\})$ and $\mathbf{X}(\varnothing) \rightarrow \mathbf{X}(\{2\})$. The Blakers-Massey theorem has been generalized in various forms to $n$-cubes by Barratt and Whitehead ([B-W]), Ellis and Steiner ([E-S]), and Goodwillie ([G2]). 
By a homotopy functor we will mean a functor from spaces to spaces which preserves weak homotopy equivalences. As a generalization to homotopy functors of the connectivity estimate provided by the Blakers-Massey theorem, Goodwillie defines what it means for a homotopy functor to satisfy stable $n$ th-order excision. Let $\mathbf{X}$ be an $n$-cube of spaces, and let $F$ be a homotopy functor. By $F(\mathbf{X})$ we mean the $n$-cube for which $(F(\mathbf{X}))(S)=F(\mathbf{X}(S))$, for $S \subseteq \underline{n}$.

Definition 1.1. $F$ is stably n-excisive with constants $c$ and $\kappa$, if, for every strongly co-Cartesian $(n+1)$-cube of spaces such that the connectivity, $k_{s}$, of $\mathbf{X}(\varnothing) \rightarrow \mathbf{X}(s)$ is at least $\kappa$ for every $s \in\{1, \ldots, n+1\}$, then $F(\mathbf{X})$ is $\left(-c+\sum_{s=1}^{n+1} k_{s}\right)$-Cartesian. We will say that $F$ satisfies $E_{n}(c, \kappa)$.

For example, the generalized form of the Blakers-Massey theorem ([G2], Theorem 2.3) states that a strongly co-Cartesian $(n+1)$-cube $\mathbf{X}$ where $\mathbf{X}(\varnothing) \rightarrow$ $\mathbf{X}(\{s\})$ is $k_{s}$-connected for each $s \in\{1, \ldots, n+1\}$ is $k$-Cartesian with $k=$ $-n+\sum_{s=1}^{n+1} k_{s}$. In other words, applying the identity functor to $\mathbf{X}$ yields a $\left(-n+\sum_{s=1}^{n+1} k_{s}\right)$-Cartesian $(n+1)$-cube. It follows that the identity functor satisfies $E_{n}(n, \kappa)$ for any $\kappa$, and hence is stably $n$-excisive. If a functor satisfies $E_{n}(c, \kappa)$ for all $c$ and $\kappa$, we say that it is $n$-excisive. That is,

Definition 1.2. $F$ is n-excisive if $F(\mathbf{X})$ is Cartesian for every strongly coCartesian $(n+1)$-cube $\mathbf{X}$.

We also have the notion of analytic functors, which are stably $n$-excisive for all $n$.

Definition 1.3. A homotopy functor $F$ is $\rho$-analytic if there is some number $q$ such that $F$ satisfies $E_{n}(n \rho-q, \rho+1)$ for all $n$.

By the above, the identity functor satisfies $E_{n}(n \rho-q, \rho+1)$ when $\rho=1$ and $q=0$. Thus, the identity functor is 1 -analytic. Other examples of analytic functors include the stable homotopy functor, $Q$, Waldhausen's functor, $A$, and the functor $X \mapsto Q(\operatorname{Map}(K, X))$ where $K$ is a fixed finite CW-complex and $\operatorname{Map}(K, X)$ is the space of all continuous maps from $K$ to $X$. For details, see section 4 of [G2]. An example of a functor which is not analytic is the functor $X \mapsto \Omega^{\infty}\left(\mathbf{E} \wedge X^{[n]}\right)$ where $\mathbf{E}$ is a spectrum that is not bounded below and $X^{[n]}$ denotes the $n$-fold smash product of $X$ with itself (see [G3], Remark 1.16).

Finally, there is a sense in which functors can be approximated by other functors.

Definition 1.4. Let $F$ and $G$ be homotopy functors. We say that $F$ and $G$ agree to order $n$ via a map $u: F \rightarrow G$ if there exist constants $c$ and $\kappa$ such that for every $\kappa$-connected space $X$, the map $u_{X}: F(X) \rightarrow G(X)$ is $(-c+(n+1) k)$-connected. We say that $u: F \rightarrow G$ satisfies $O_{n}(c, \kappa)$.

In [G3], Goodwillie provides a method by which an analytic functor can be approximated by a tower of excisive functors. The idea is that a stably $n$ excisive functor $F$ can be approximated by an $n$-excisive functor $P_{n} F . P_{n} F$ can be constructed along with a transformation $p_{n} F: F \rightarrow P_{n} F$ such that $P_{n} F$ is $n$-excisive and $F$ agrees with $P_{n} F$ to order $n$ via $p_{n} F . P_{n} F$ is regarded as an " $n$th degree Taylor polynomial" approximation to $F$. If $F$ is 
$\rho$-analytic, then it follows that there is an entire sequence of functors $P_{0} F=$ $F(*), P_{1} F, \ldots, P_{n-1} F, P_{n} F, \ldots$ such that each $P_{k} F$ is $k$-excisive and agrees with $F$ to order $k$. Furthermore, there are maps $q_{n} F: P_{n} F \rightarrow P_{n-1} F$ such that these functors fit together into a tower of functors. This tower is called the Taylor tower of $F$. More formally, we have the following results.

Theorem 1.5 (Goodwillie). Let $F$ be a $\rho$-analytic functor. To any basepointed space $X$ there are naturally associated objects $P_{n} F(X)$ and maps $p_{n} F$ and $q_{n} F$ which fit together in a tower:

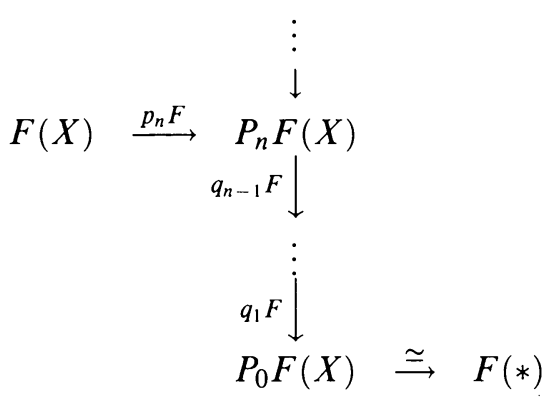

which satisfies $\left(q_{n} F\right) \circ\left(p_{n} F\right)=p_{n-1} F . P_{n} F$ is an n-excisive functor. If $X$ is $(\rho+1)$-connected then the connectivity of the map $p_{n} F: F(X) \rightarrow P_{n} F(X)$ tends to $+\infty$ with $n . F(X)$ is equivalent to the homotopy limit, $P_{\infty} F(X)$, of the tower.

For the construction of $P_{n} F, p_{n} F$, and $q_{n} F$ see section 1 of [G3].

The $n$th layer of the Taylor tower of an analytic functor $F$ is

$$
D_{n} F=\operatorname{fiber}\left(P_{n} F \rightarrow P_{n-1} F\right) .
$$

Here and elsewhere, fiber will mean the homotopy fiber. Each layer of the Taylor tower is a functor of a special form, resembling a monomial of degree $n$. Specifically, it is a homogeneous functor of degree $n$, as we state below.

Definition 1.6. A homotopy functor $F$ is homogeneous of degree $n$ if it is $n$-excisive and $P_{n-1} F \simeq *$.

Goodwillie provides a classification of all such functors in [G3]. The result is stated below. By homotopy orbit spectrum we mean the spectrum obtained from a spectrum with $G$-action (for some finite group $G$ ) by taking the homotopy orbit space $\left(X_{n} \wedge_{G} E G_{+}\right)$of each space, $X_{n}$, in the spectrum.

Theorem 1.7. If $F$ is homogeneous of degree $n$ and $X$ is a space, then

$$
F(X) \simeq \Omega^{\infty}\left(\mathbf{C} \wedge X^{[n]}\right) h \Sigma_{n}
$$

where $\mathbf{C}$ is a spectrum with $\Sigma_{n}$-action, $\Sigma_{n}$ is the symmetric group on $n$ letters, $X^{[n]}$ is the $n$-fold smash product of $X$ with itself, and $h \Sigma_{n}$ denotes the homotopy orbit spectrum.

As claimed, we have the following result.

Proposition 1.8. If $F$ is an analytic functor, then $D_{n} F$ is homogeneous of degree $n$. 
It follows that $D_{n} F(X)$ has the form $\Omega^{\infty}\left(\mathbf{C} \wedge X^{[n]}\right)$ for some spectrum, C, with $\Sigma_{n}$-action. $\mathrm{C}$ is regarded as the "coefficient" of $D_{n} F$. As we will see later, it is also the $n$th derivative of $F$ at a point.

\section{THE IDENTITY FUNCTOR}

In this section, we describe the problem motivating this paper, and outline the results obtained thus far. Our object of study is the identity functor of topological spaces: the functor from the category of topological spaces to itself which takes a space to itself. This functor will be denoted by $I$. The goal is to determine the Taylor tower of $I$. As we saw in section 1 , the generalized Blakers-Massey theorem tells us that $I$ is a 1 -analytic functor. $I$ does not have finite degree, i.e., it is not homogeneous of degree $n$, nor does its Taylor tower split as a product of functors .

The Taylor tower of $I$ is of interest because of the information it will provide about homotopy theory. The fact that $I$ is 1 -analytic means that its Taylor tower converges on 2-connected spaces. That is, each finite stage of the tower yields, for a $k$-connected space $X$, another space $P_{n} I(X)$ whose first $(n+1) k$ homotopy groups are the same as those of $X$. Hence, as $n$ increases, $P_{n} I(X)$ approximates the homotopy of $X$ in a greater and greater range. Goodwillie has also pointed out that there is a spectral sequence that converges to the homotopy groups of $X$ in which the $E^{2}$ terms are given by the homotopy groups of the $D_{n} I(X)$ 's . Before this spectral sequence can be utilized, the maps between the $D_{n} I$ 's must be determined, i.e., we need to know how the individual layers of the Taylor tower fit together within the tower.

If we look at convergence on the level of functors rather than spaces then we see that the Taylor tower is a sequence of functors which link stable and unstable homotopy theory. Specifically, the first layer of the tower is the stable homotopy functor $Q$. Considering the tower as converging to the "unstable homotopy functor" $I$, we see that each stage of the tower, $P_{n} I$, recovers increasingly more information about unstable homotopy theory, information which was lost when $Q$ was applied. This tower should yield new ideas about the relationship between stable and unstable homotopy theory.

At this time, the Taylor tower of $I$ has not been determined. In this paper, we complete a first step in the problem, that of determining the derivatives of $I$ at a point. Goodwillie has previously determined the first two derivatives of $I$ and the homogeneous degree $n$ functors in [G1] and [G3]. Specifically, he has shown the following

\section{Proposition 2.1.}

(a) The first derivative of $I$ is the sphere spectrum $\mathbf{S}^{0}$.

(b) The second derivative of I is the (-1)-sphere spectrum, $\mathbf{S}^{-1}$, with trivial $\Sigma_{2}$-action.

(c) $D_{1} I(X) \simeq Q(X)$ for any 2-connected space $X$.

(d) $D_{2} I(X) \simeq \Omega Q\left((X \wedge X)_{h \Sigma_{2}}\right)$ for any 2-connected space $X$.

The homotopy type of the derivatives have also been known for a while. The homotopy types can be determined by the Hilton-Milnor theorem (see section 5 ). The elusive part of the problem has been to determine the $\Sigma_{n}$-action on the spectrum. One solution is given by John Rognes in his dissertation [R]. In 
it he computes the first nontrivial homotopy group of the cross effect functor of $\Omega^{l} \Sigma^{l}$. From this the homotopy of the derivatives of $I$ with $\Sigma_{n}$-action can be recovered. He accomplishes this by identifying the homotopy group as the kernel of a map from $\pi_{*}\left(\Omega^{l} \Sigma^{l}\left(X_{1} \vee X_{2} \vee \cdots \vee X_{n}\right)\right)$ to

$$
\prod_{k=1}^{n} \pi_{*}\left(\Omega^{l} \Sigma^{l}\left(X_{1} \vee X_{2} \vee \cdots \vee \widehat{X_{k}} \vee \cdots \vee X_{n}\right)\right)
$$

where each component of the map is a collapsing map $\bigvee_{i=1}^{n} X_{i} \rightarrow\left(X_{1} \vee X_{2} \vee \cdots \vee\right.$ $\left.\widehat{X_{k}} \vee \cdots \vee X_{n}\right)$. He then compares the kernel of the map of homotopy groups with the kernel of the same map in homology, and uses the Snaith splitting to identify the kernel in homology as the homology of a configuration space smashed with $X_{1} \wedge X_{2} \wedge \cdots \wedge X_{n}$. The final step is to show that the Hurewicz map between the kernel in homology and the kernel in homotopy is a $\Sigma_{n}$-isomorphism. This is done by means of the Browder operations on homology. The homology of the configuration space in the kernel is calculated by finding the homology of a related quotient complex of the standard $\left(\begin{array}{l}n \\ 2\end{array}\right)$-simplex.

This paper computes the derivatives in a more direct fashion, working with spaces rather than homotopy and homology groups, and avoids the need for the Snaith splitting or the Browder operations. We construct a new symmetric functor on $n$ spaces defined by

$$
M_{n}\left(X_{1}, X_{2}, \ldots, X_{n}\right)=\operatorname{Map}_{*}\left(\Delta_{n}, X_{1} \wedge X_{2} \wedge \cdots \wedge X_{n}\right)
$$

where $\operatorname{Map}_{*}$ denotes basepointed maps and $\Delta_{n}$ is a quotient space of the product of $n$ copies of the $(n-1)$-cube. $\Delta_{n}$ has the same homotopy type as the wedge of $(n-1)$ ! copies of the $(n-1)$-sphere. $M_{n}$ will be related by Theorem 2.2 below to the $n$th cross effect of $I, \chi_{n} I$, a symmetric functor of $n$ variables defined in section 3 (Definition 3.4). As will be shown in Proposition 4.1 and its corollary, Theorem 2.2 guarantees that $M_{n}$ satisfies conditions necessary for its multilinearization to be equivalent to that of $\chi_{n} I$. In turn, Proposition 3.13 will establish the relationship between the multilinearization of $\chi_{n} I$ and the $n$th derivative of $I$. The main result is the following.

Theorem 2.2. There is a natural transformation of symmetric functors:

$$
T: \chi_{n} I\left(X_{1}, X_{2}, \ldots, X_{n}\right) \rightarrow \operatorname{Map}_{*}\left(\Delta_{n}, X_{1} \wedge X_{2} \wedge \cdots \wedge X_{n}\right)
$$

which satisfies the following properties:

(a) $T$ is $\Sigma_{n}$-equivariant, that is, it preserves the $\Sigma_{n}$-symmetry of $\chi_{n} I$ and $M_{n}$ which permutes the spaces $X_{1}, X_{2}, \ldots, X_{n}$ and images of the $(n-1)$-cubes in $\Delta_{n}$.

(b) If $X_{1}, X_{2}, \ldots, X_{n}$ are $k$-connected then

$$
\begin{aligned}
& \Omega T_{n}: \Omega \chi_{n} I\left(\Sigma X_{1}, \Sigma X_{2}, \ldots, \Sigma X_{n}\right) \rightarrow \Omega \mathrm{Map}_{*}\left(\Delta_{n}, \Sigma X_{1} \wedge \Sigma X_{2} \wedge \cdots \wedge \Sigma X_{n}\right) \\
& \quad \text { is }(n+1)(k+1)-1 \text {-connected. }
\end{aligned}
$$

From Theorem 2.2 it easily follows that:

\section{Corollary 2.3.}

(a) The nth derivative of $I$, denoted $I^{(n)}$, is the spectrum whose $k$ th term is $\operatorname{Map}_{*}\left(\Delta_{n}, \Sigma^{k}\right)$. This spectrum has the obvious $\Sigma_{n}$-action given by permuting the $(n-1)$-cubes of $\Delta_{n}$. 
(b) The homotopy type of $I^{(n)}$ is the same as that of the wedge of $(n-1)$ ! copies of the $(1-n)$-sphere spectrum.

The proofs of Theorem 2.2 and Corollary 2.3 will be given in the subsequent sections. In section 3 we will describe the general method for calculating derivatives of homotopy functors. In section 4 we will construct the quotient space $\Delta_{n}$ and the transformation $T_{n}$ and establish the $\Sigma_{n}$-equivariance of $T$. In section 5 we will determine the homotopy type of the $\Delta_{n}$ (and consequently, of $I^{(n)}$ ), and section 6 will be devoted to proving Theorem $2.2 \mathrm{~b}$.

\section{THE $n$ TH DERIVATIVE OF A FUNCTOR}

In this section we will define the $n$th derivative of a homotopy functor and show how it can be calculated in general. In the traditional calculus of realvalued functions one first defines the derivatives of a function and then uses these to construct the Taylor series of the function. In the case of homotopy functors, the opposite is true. It is more natural to define the Taylor tower of a functor first and then define the derivatives of the functor as the coefficients of its Taylor tower. Recall, the $n$th layer of the Taylor tower of a functor $F$ is $D_{n} F=$ fiber $\left(P_{n} F \rightarrow P_{n-1} F\right)$. By Proposition 1.8 , if $F$ is analytic, $D_{n} F$ is a homogeneous functor of degree $n$. We saw in Theorem 1.7 that such a functor is naturally equivalent to a functor of the form

$$
G(X)=\Omega^{\infty}\left(\mathbf{C} \wedge X^{[n]}\right)_{h \Sigma_{n}}
$$

where $\mathbf{C}$ is a spectrum with $\Sigma_{n}$-action, $X^{[n]}$ is the $n$-fold smash product of $X$ and $h \Sigma_{n}$ denotes the homotopy orbit spectrum. It is the spectrum that is the coefficient of the homogeneous degree $n$ functor that Goodwillie defines to be the $n$th derivative of $X$.

One does not need to know $P_{n} I$ and $P_{n-1} I$ to determine the $n$th derivative of $I$. We will work with another category of functors which are equivalent to homogeneous functors of degree $n$, namely symmetric multilinear functors. These functors will be defined and discussed in this section. Symmetric multilinear functors are functors of several variables and, as we will see in Proposition 3.11 , are also equivalent to functors of the form

$$
G\left(X_{1}, \ldots, X_{n}\right)=\Omega^{\infty}\left(\mathbf{C} \wedge X_{1} \wedge \cdots \wedge X_{n}\right)
$$

where $\mathbf{C}$ is a spectrum with $\Sigma_{n}$-action. In defining the $n$th derivative we will identify, without needing to know $P_{n} I$ or $P_{n-1} I$, a symmetric multilinear functor which is naturally equivalent to $D_{n} I$ and determine the $n$th derivative from this functor. This symmetric multilinear functor is the multilinearization of the $n$th cross effect functor of $I$ and is called the $n$th differential of $I$. This section will be devoted to explicitly defining the derivative as outlined above. The treatment will consist of three parts: defining the cross effect functor, discussing multilinearization and symmetric multilinear functors, and defining the derivative. This process is the way in which the derivative is originally defined by Goodwillie. Most of the material can be found in section 1 of [G1] and sections 3 and 4 of [G3].

To begin, we will let $F$ be a homotopy functor from the category of based spaces to itself. We will construct its $n$th cross effect. The $n$th cross effect is the total fiber of a particular $n$-cube of spaces. The first step in defining the $n$th cross effect of $F$ is to define the total fiber of an $n$-cube of spaces. 
Definition 3.1. The total fiber of an $n$-cube of spaces $\mathbf{X}$, denoted $\tilde{f} \mathbf{X}$, is the homotopy fiber of the map $\gamma: \mathbf{X}(\varnothing) \rightarrow \operatorname{holim}_{U \in N, U \neq \varnothing}(\mathbf{X}(U))$.

We may also define the total fiber of an $n$-cube of spaces inductively.

Remark 3.2. For a 1-cube (i.e., a map of spaces)

$$
f: X \rightarrow Y
$$

the total fiber is just the homotopy fiber of $f$.

For an $n$-cube of spaces, $\mathbf{X}$, we can consider $\mathbf{X}$ as a map of $(n-1)$-cubes:

$$
\mathbf{Y} \rightarrow \mathbf{Z}
$$

We can then define the total fiber of $\mathbf{X}$ inductively as

$$
\tilde{f} \mathbf{X}=\operatorname{fiber}(\tilde{f} \mathbf{Y} \rightarrow \tilde{f} \mathbf{Z}) .
$$

That this inductive definition of the iterated homotopy fiber is equivalent to Definition 3.1 follows from properties of the homotopy inverse limit. (See [G2].)

We will need the following $n$-cube of spaces associated to $F$ in order to define the $n$th cross effect of $F$.

Definition 3.3. Given a collection of $n$ spaces $\mathbf{X}=\left\{X_{1}, \ldots, X_{n}\right\}, F_{\mathbf{X}}$ is the $n$-cube of spaces defined by $F_{\mathbf{X}}(\{1, \ldots, n\})=F(*)$ and $F_{\mathbf{X}}(U)=F\left(\bigvee_{i \notin U} X_{i}\right)$ when $U \neq\{1, \ldots, n\}$. If $U \subset V$ then the morphism $F_{\mathbf{X}}(U \rightarrow V)$ is $F\left(g_{U, V}\right)$ where $g_{U, V}$ is the retraction of $\bigvee_{i \notin U} X_{i}$ to $\bigvee_{j \notin V} X_{j}$, collapsing any $X_{i}$ such that $i \in V$ and $i \notin U$ to the basepoint.

For example, if we have $\mathbf{X}=\left\{X_{1}, X_{2}\right\}$ then $F_{\mathbf{X}}$ is the square below.

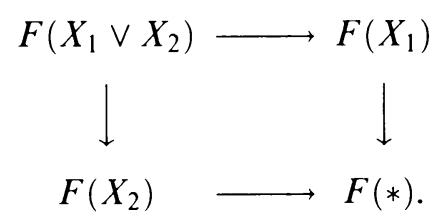

Now we can define the cross effect.

Definition 3.4. The nth cross effect of a functor $F$ evaluated at the spaces $\left\{X_{1}, \ldots, X_{n}\right\}$ is a functor of $n$ variables defined as the total fiber of the $n$ cube $F_{\mathbf{X}}$ with $\mathbf{X}=\left\{X_{1}, \ldots, X_{n}\right\}$. The cross effect will be denoted $\chi_{n} F$.

$\chi_{n} F$ is a symmetric functor, where a symmetric functor is defined as follows.

Definition 3.5. A homotopy functor $F$ is called symmetric if for each $\pi \in \Sigma_{n}$ and spaces $X_{1}, \ldots, X_{n}$ there is an isomorphism

$$
F_{\pi}: F\left(X_{1}, \ldots, X_{n}\right) \rightarrow F\left(X_{\pi(1)}, \ldots, X_{\pi(n)}\right)
$$

and for every $\pi, \sigma \in \Sigma_{n}$

$$
F_{\sigma \circ \pi}=F_{\pi} \circ F_{\sigma} .
$$

That $\chi_{n} F$ is symmetric is clear from the symmetry of the $n$-cube with respect to the spaces $X_{1}, \ldots, X_{n}$.

Now that we have defined the cross effect of $F$, we must explain what it means to multilinearize the cross effect, or to linearize a functor in general. The 
multilinearization of $\chi_{n} F$ will be the symmetric multilinear functor equivalent to $D_{n} F$ mentioned at the beginning of the section. First, we review the concept of linear functors. The process of linearization of a functor $F$ is basically that of finding the linear functor which most closely agrees with $F$.

Definition 3.6. A homotopy functor $F$ is called linear if it satisfies the properties below.

(a) $F$ is reduced, i.e., $F(*)$ is contractible.

(b) $F$ is 1-excisive.

For a discussion of linear functors see section 1 of [G1] or chapter 4 of [J].

To any functor $F$ satisfying stable first order excision (recall Definition 1.1) we may also associate a linear functor. As stated in section $1, P_{1} F$ is the degree one (or excisive) functor which most closely approximates $F$. We make $P_{1} F$ a linear functor by reducing it, i.e., by taking the homotopy fiber of $P_{1} F(X) \rightarrow$ $P_{1} F(*)$. If the functor is already reduced, then the process of linearizing it can be made even more explicit. $P_{1} F$ is reduced and hence is already linear. We have:

Proposition 3.7. If $F$ is a reduced homotopy functor satisfying stable first order excision then the linearization of $F$ is simply $P_{1} F$ and is given by

$$
P_{1} F(X)=\underset{n}{\operatorname{hocolim}} \Omega^{n} F\left(\Sigma^{n} X\right) \text {. }
$$

Proof. The proof of this proposition follows directly from the definition of $P_{1} F$ that Goodwillie gives in [G1]. Specifically, $P_{1} F$ is defined as the homotopy colimit of the diagram

$$
F(X) \rightarrow T F(X) \rightarrow T(T F(X)) \rightarrow \ldots
$$

where

$$
T F(X)=\operatorname{holim}(F(C X) \rightarrow F(\Sigma X) \leftarrow F(C X)) .
$$

$C X$ is the cone on $X$. When $F$ is reduced, we see that $T F(X)$ is equivalent to $\Omega F \Sigma X$, and the result follows.

Proposition 3.7 will be sufficient for our purposes since we will only need to linearize reduced functors.

If we have a functor of $n$ variables, $G$, we may speak of its multilinearization. The multilinearization of $G$ is simply the linearization of $G$ with respect to each of its variables. That is, we hold all but one variable fixed and linearize $G$ with respect to the unfixed variable and then repeat this process for each variable. As in the case of functors of one variable, if $G$ is a reduced functor with respect to each of its variables, then we can give the multilinearization of $G$ explicitly. This is a direct consequence of Proposition 3.7.

Corollary 3.8. If $G$ is a homotopy functor of $n$ variables which satisfies first order excision and is reduced with respect to each of its variables, then the multilinearization of $G$ is given by

$$
\widetilde{G}\left(X_{1}, \ldots, X_{n}\right)=\underset{k_{1}, \ldots, k_{n}}{\operatorname{hocolim}} \Omega^{k_{1}+\cdots+k_{n}}(G)\left(\Sigma^{k_{1}} X_{1}, \ldots, \Sigma^{k_{n}} X_{n}\right) .
$$

A functor which has been multilinearized or is linear in each of its variables is called a multilinear functor.

With the preceding definitions, we are now able to define the $n t h$ differential of a homotopy functor . 
Definition 3.9. The $n$th differential of a homotopy functor $F$, denoted $D^{(n)} F$, is the functor of $n$ variables defined by

$$
D^{(n)} F\left(X_{1}, \ldots, X_{n}\right)=\widetilde{\chi_{n} F}\left(X_{1}, \ldots, X_{n}\right) .
$$

That is, $D^{(n)} F$ is the multilinearization of the $n$th cross effect of $F$.

$D^{(n)} F$ is an example of a symmetric multilinear functor.

Definition 3.10. A symmetric multilinear functor of $n$ variables is a symmetric functor which is linear in each of its variables.

That $D^{(n)} F$ is a symmetric functor follows from the symmetry of the cross effect already established. Its multilinearity follows from the definition.

As stated at the beginning of the section, symmetric multilinear functors are naturally equivalent to spectra with $\Sigma_{n}$-action. Precisely, Goodwillie has shown (in [G3])

Proposition 3.11. If $G$ is a symmetric multilinear functor of $n$ variables then it has the following form:

$$
G\left(X_{1}, \ldots, X_{n}\right)=\Omega^{\infty}\left(\mathbf{C} \wedge X_{1} \wedge \cdots \wedge X_{n}\right)
$$

where $\mathbf{C}$ is a spectrum with $\Sigma_{n}$-action.

With this proposition we are now able to define the $n$th derivative of the functor $F$.

Definition 3.12. The $n t h$ derivative of the homotopy functor $F$ is the spectrum with $\Sigma_{n}$-action associated with the symmetric multilinear functor $D^{(n)} F$, that is, the spectrum $\mathbf{C}$ such that

$$
D^{(n)} F\left(X_{1}, \ldots, X_{n}\right) \simeq \Omega^{\infty}\left(\mathbf{C} \wedge X_{1} \wedge \cdots \wedge X_{n}\right) .
$$

We write $F^{(n)}=\mathbf{C}$.

We should note here that the definition of the derivative given above is the $n$th derivative of the functor $F$ at a point. A more general definition can be given for derivatives at any space $X$. (See [G3].) For examples of derivatives, see $[\mathrm{G} 1]$ and [G3].

We conclude this section by justifying the analogy drawn at the beginning of the section between the $n$th derivative of $F$ as we have defined it and the coefficient of the $n$th term in the Taylor tower of $F$. The $n$th layer of the Taylor tower of $F, D_{n} F$, is a homogeneous functor of degree $n$. We stated that a homogeneous functor of degree $n$ such as $D_{n} F$ was naturally equivalent to a symmetric multilinear functor and could be written in the form

$$
\Omega^{\infty}\left(\mathbf{C} \wedge X^{[n]}\right)_{h \Sigma_{n}}
$$

where $\mathbf{C}$ is a spectrum with $\Sigma_{n}$-action. Under the equivalence that Goodwillie establishes between homogeneous functors of degree $n$, symmetric multilinear functors and spectra with $\Sigma_{n}$-action, $D^{(n)} F$ is the symmetric multilinear functor equivalent to $D_{n} F$ and $F^{(n)}$ is the spectra with $\Sigma_{n}$-action equivalent to $D_{n} F$. This is summarized below. 


\section{Proposition 3.13.}

(a) The symmetric multilinear functor equivalent to $D_{n} F$ is the cross effect of $D_{n} F, \chi_{n} D_{n} F$. Furthermore, this functor is naturally equivalent to the multilinearization of $\chi_{n} F$.

(b) $D_{n} F$ can be recovered from $F^{(n)}$ :

$$
D_{n} F(X)=\left(F^{(n)} \wedge X^{[n]}\right)_{h \Sigma_{n}} .
$$

This process can be extended to all homogeneous functors of degree $n$ to give an explicit means of constructing the equivalences between the three categories. See [G3], section 3 for details.

We are now ready to construct the derivatives of $I$.

\section{THE FUNCTOR $M_{n}$}

This section will be devoted to the construction of the functor $M_{n}$ and the transformation $T$ between $\chi_{n} I$ and $M_{n}$, first mentioned in section 2 . We will show later that the multilinearization of $M_{n}$ is equivalent to the multilinearization of $\chi_{n} I$, and from that determine the $n$th derivative of $I$.

Recall Theorem 2.2. Conditions (a) and (b) are sufficient to conclude that $\chi_{n} I$ and $M_{n}$ have the same multilinearization. Specifically, we have

Proposition 4.1. Let $F$ and $G$ be reduced functors of $n$ variables. Let $S: F \rightarrow$ $G$ be a natural transformation such that when $X_{1}, X_{2}, \ldots, X_{n}$ are $k$-connected, then the resulting map

$$
S: F\left(X_{1}, X_{2}, \ldots, X_{n}\right) \rightarrow G\left(X_{1}, X_{2}, \ldots, X_{n}\right)
$$

is $((n+1) k-c)$-connected, where $c$ is a constant which does not depend on $k$. ( $F$ and $G$ agree to nth order via $S$.) If such a transformation exists, then the multilinearization of $F$ is equivalent to the multilinearization of $G$.

Proof. The multilinearizations of $F$ and $G$ evaluated at the spaces $X_{1}, \ldots$, $X_{n}$ are equivalent to hocolim $\operatorname{lom}_{l \rightarrow \infty} \Omega^{l n} F\left(\Sigma^{l} X_{1}, \Sigma^{l} X_{2}, \ldots, \Sigma^{l} X_{n}\right)$ and hocolim $_{l \rightarrow \infty} \Omega^{l n} G\left(\Sigma^{l} X_{1}, \Sigma^{l} X_{2}, \ldots, \Sigma^{l} X_{n}\right)$, respectively. If $X_{1}, \ldots, X_{n}$ are $k$ connected then $\Sigma^{l} X_{1}, \Sigma^{l} X_{2}, \ldots, \Sigma^{l} X_{n}$ are $(k+l)$-connected. Hence, the map

$$
\Omega^{l n} S: \Omega^{l n} F\left(\Sigma^{l} X_{1}, \ldots, \Sigma^{l} X_{n}\right) \rightarrow \Omega^{l n} G\left(\Sigma^{l} X_{1}, \ldots, \Sigma^{l} X_{n}\right)
$$

is $((n+1)(k+l)-c-l n)=n k+k+l-c$-connected. As $l$ goes to infinity, so does the connectivity. Hence, in the limit, $\Omega^{l n} F\left(\Sigma^{l} X_{1}, \ldots, \Sigma^{l} X_{n}\right)$ and $\Omega^{\ln } G\left(\Sigma^{l} X_{1}, \ldots, \Sigma^{l} X_{n}\right)$ are equivalent.

Corollary 4.2. If $\Omega T_{n}: \Omega \chi_{n} I\left(\Sigma X_{1}, \ldots, \Sigma X_{n}\right) \rightarrow \Omega \operatorname{Map}_{*}\left(\Delta_{n}, \Sigma X_{1} \wedge \cdots \wedge \Sigma X_{n}\right)$ is $(n+1) k-c$-connected then

$$
\chi_{n} I\left(X_{1}, X_{2}, \ldots, X_{n}\right) \rightarrow M_{n}\left(X_{1}, X_{2}, \ldots, X_{n}\right)
$$

is an equivalence after multilinearization.

Proof. The hypothesis implies that the map

$$
\Omega^{n} T_{n}: \Omega^{n} \chi_{n} I\left(\Sigma X_{1}, \ldots, \Sigma X_{n}\right) \rightarrow \Omega^{n} \operatorname{Map}_{*}\left(\Delta_{n}, \Sigma X_{1} \wedge \cdots \wedge \Sigma X_{n}\right)
$$

is $((n+1) k-(n-1)-c)$-connected. Proposition 4.1 implies that the multilinearizations of each side above are equivalent. But the multilinearizations 
of $\chi_{n} I$ and $M_{n}$ are equivalent to those of $\Omega^{n} \chi_{n} I\left(\Sigma X_{1}, \ldots, \Sigma X_{n}\right)$ and $\Omega^{n} \operatorname{Map}_{*}\left(\Delta_{n}, \Sigma X_{1} \wedge \cdots \wedge \Sigma X_{n}\right)$ respectively. Hence, the multilinearizations of $\chi_{n} I$ and $M_{n}$ are equivalent.

Hence, condition (b) of Theorem 2.2 guarantees that the spectra associated to the multilinearizations of $\chi_{n} I$ and $M_{n}$ have the same homotopy type. Condition (a) of Theorem 2.2 ensures that the spectra have the same $\Sigma_{n}$-action. Thus, if we can prove Theorem 2.2 , we will be able to recover the derivatives of $I$ from the multilinearization of $M_{n}$. In this section we will define $T$ and $\Delta_{n}$. We will also verify condition (a) of Theorem 2.2.

If we use the inductive definition (Remark 3.2) to construct the total fiber of an $n$-cubical diagram, we see that the resulting space is equivalent to a collection of maps with certain compatibility properties. For example, the iterated fiber of a 1-cube, $f: X \rightarrow Y$, is, by definition,

$$
\left\{(x, \gamma) \in X \times Y^{I} \mid \gamma(0)=f(x), \gamma(1)=y_{0}\right\}
$$

where $y_{0}$ is the basepoint in $Y$. The exact description for any $n$-cube is given below in Definition 4.3, following remarks in section 0 of [G2]. This property of the total fiber will be used in constructing $T$ and $\Delta_{n}$.

Definition 4.3. Let $\mathbf{X}$ be an $n$-cube of spaces. For a subset $U$ of $\underline{n}$, let $I^{U}=$ $\left\{\left(t_{1}, t_{2}, \ldots, t_{n}\right) \in I^{n} \mid t_{i}=0\right.$ if $\left.i \notin U\right\}$. A point in the total fiber of $\mathbf{X}$ is a collection of maps, $\Phi=\left\{\Phi_{U}\right\}_{U \subset n}$, where $\Phi_{U}: I^{U} \rightarrow \mathbf{X}(U)$. Furthermore, these maps must satisfy the compatibility properties below:

(a) For $V \subset U$

$$
\left.\Phi_{U}\right|_{I^{r}}=\gamma_{V, U} \circ \Phi_{V}
$$

where $\gamma_{V, U}$ is the map $\mathbf{X}\left(i_{V, U}\right): \mathbf{X}(V) \rightarrow \mathbf{X}(U)$, and $i_{V, U}$ is the inclusion $V \hookrightarrow U$.

(b) $\Phi_{U}\left(t_{1}, \ldots, t_{n}\right)$ is the basepoint in $\mathbf{X}(U)$ if $t_{i}=1$ for some $i$.

Using 4.3 we will begin constructing $T$. First we show how to construct a map $T_{n}^{\prime}: \chi_{n} I\left(X_{1}, X_{2}, \ldots, X_{n}\right) \rightarrow \operatorname{Map}_{*}\left(I^{n(n-1)}, \prod_{i=1}^{n} X_{i}\right)$. Then we compose with the quotient map from the product $\prod_{i=1}^{n} X_{i}$ to the smash product $\bigwedge_{i=1}^{n} X_{i}$. This gives us a map from $\chi_{n} I\left(X_{1}, X_{2}, \ldots, X_{n}\right)$ to $\operatorname{Map}_{*}\left(I^{n(n-1)}, \bigwedge_{i=1}^{n} X_{i}\right)$. Finally, we will identify a subspace of $I^{n(n-1)}$ which is always mapped to the basepoint in the smash product. Taking the quotient of $I^{n(n-1)}$ over this subspace yields the space we will call $\Delta_{n}$ and allows us to define the functor $M_{n}$ as $\operatorname{Map}_{*}\left(\Delta_{n}, \bigwedge_{i=1}^{n} X_{i}\right)$. Furthermore, this yields the desired map from the cross effect to $M_{n}, T_{n}: \chi_{n} I\left(X_{1}, X_{2}, \ldots, X_{n}\right) \rightarrow \operatorname{Map}_{*}\left(\Delta_{n}, \bigwedge_{i=1}^{n} X_{i}\right)$.

If we apply Definition 4.3 to $\chi_{n} I\left(X_{1}, X_{2}, \ldots, X_{n}\right)$ then we see that a point $\Phi$ in $\chi_{n} I$ consists of maps $\left\{\Phi_{U}\right\}_{U \subset\{1, \ldots, n\}}$ such that

$$
\Phi_{U}: I^{U} \rightarrow \bigvee_{i \notin U} X_{i}
$$

and conditions (a) and (b) of 4.3 are satisfied.

Consider the set $U_{i}=\{1,2, \ldots, \hat{i}, \ldots, n\}$. We have

$$
\Phi_{U_{1}}: I^{U_{\imath}} \rightarrow X_{i} .
$$


Note that $I^{U_{i}}$ is an $(n-1)$-cube. Let

$$
T_{n}^{\prime}: \chi_{n} I\left(X_{1}, X_{2}, \ldots, X_{n}\right) \rightarrow \operatorname{Map}_{*}\left(I^{n(n-1)}, \prod_{i=1}^{n} X_{i}\right)
$$

be defined by

$$
T_{n}^{\prime}(\Phi)=\prod_{i=1}^{n} \Phi_{U_{i}}: \prod_{i=1}^{n} I^{U_{i}} \rightarrow \prod_{i=1}^{n} X_{i} .
$$

If we compose with the quotient map from $\prod_{i=1}^{n} X_{i}$ to $\bigwedge_{i=1}^{n} X_{i}$, then we have

$$
T_{n}^{\prime \prime}: \chi_{n} I\left(X_{1}, X_{2}, \ldots, X_{n}\right) \rightarrow \operatorname{Map}_{*}\left(I^{n(n-1)}, \bigwedge_{i=1}^{n} X_{i}\right)
$$

defined by

$$
T_{n}^{\prime \prime}(\Phi)=\bigwedge_{i=1}^{n} \tau_{i}
$$

Since we have now passed to the smash product, there will be many more points in $I^{n(n-1)}$ mapped by $T_{n}^{\prime \prime}(\Phi)$ to the basepoint in the new target $\bigwedge_{i=1}^{n} X_{i}$. Specifically, if $T_{n}^{\prime}(\Phi)(t) \in\left(X_{j} \vee X_{k}\right) \times\left(\prod_{i=1, i \neq j, k}^{n} X_{i}\right)$ for some $j$ and $k$, then $T_{n}^{\prime \prime}(\Phi)(t)$ is the basepoint in $\bigwedge_{i=1}^{n} X_{i}$. With this in mind, recall properties (a) and (b) of Definition 4.3. These properties tell us that if we restrict $\Phi_{U_{i}}$ to the boundary of $I^{U_{i}}$ then either a point on the boundary is mapped by $\Phi_{U_{i}}$ to the basepoint in $X_{i}$ (if one of the coordinates of the point is 1) or its image under $\Phi_{U_{i}}$ is the image under the retraction $X_{i} \vee X_{j} \rightarrow X_{i}$ of a point in $X_{i} \vee X_{j}$, for some $j$ (if one of the coordinates of the boundary point is 0 ). Knowing this, we can identify a collection of subspaces of $I^{n(n-1)}$ which will always be mapped to the basepoint in $\bigwedge_{i=1}^{n} X_{i}$ by $T_{n}^{\prime \prime}$.

Let $t=\left[t_{i j}\right]_{1 \leq i, j \leq n}$ where $t_{i i}=0$ for all $i$ denote a point in $I^{n(n-1)}$, so that $T^{\prime \prime}(\Phi)$ maps the $(n-1)$-cube, $\left\{\left(t_{i 1}, t_{i 2}, t_{i 3}, \ldots, t_{i i-1}, 0, t_{i i+1}, \ldots, t_{i n}\right)\right\}$, to $X_{i}$. We wish to consider the following subspaces of $I^{n(n-1)}$.

Definition 4.4. For $1 \leq i<j \leq n$, let $W_{i j}=\left\{t \in I^{n(n-1)} \mid t_{i k}=t_{j k}\right.$ for $1 \leq k \leq$ $n\}$.

As indicated, if one of the coordinates, $t_{i j}$, of $t \in I^{n(n-1)}$ is 0 , then $\Phi_{U_{i}}\left(t_{i 1}, \ldots, t_{i n}\right)$ is the retraction to $X_{i}$ of a point in $X_{i} \vee X_{j}$. The conditions placed on a point in $W_{i j}$ guarantee that $\Phi_{U_{i}}\left(t_{i 1}, \ldots, t_{i n}\right)$ and $\Phi_{U_{j}}\left(t_{j 1}, \ldots, t_{j n}\right)$ are retractions to $X_{i}$ and $X_{j}$ respectively of the same point in $X_{i} \vee X_{j}$. Hence, we state the following.

Lemma 4.5. If $t \in W_{i j}$, then $T_{n}^{\prime \prime}(\Phi)(t)=e$, the basepoint in $\bigwedge_{i=1}^{n} X_{i}$.

Proof. Let $t=\left[t_{k l}\right] \in W_{i j}$. The essence of the proof was described before the statement of the lemma. By property (a) of Definition 4.3 we have

$$
\left.\Phi_{U_{i}}\right|_{I^{V}}=\gamma_{V, U_{i}} \circ \Phi_{V}
$$

and

$$
\left.\boldsymbol{\Phi}_{U_{j}}\right|_{I^{\nu}}=\gamma_{V, U_{j}} \circ \boldsymbol{\Phi}_{V}
$$

where $V=\{1, \ldots, \hat{i}, \ldots, \hat{j}, \ldots, n\}$. For a point $t \in W_{i j}$, we see that

$$
\boldsymbol{\Phi}_{U_{i}} \times \boldsymbol{\Phi}_{U_{j}}\left(\left(t_{i 1}, \ldots, t_{i n}\right),\left(t_{j 1}, \ldots, t_{j n}\right)\right)
$$


is equal to

$$
\left(\gamma_{V, U_{i}} \circ \Phi_{V}\left(t_{i 1}, \ldots, t_{i n}\right), \gamma_{V, U_{j}} \circ \Phi_{V}\left(t_{i 1}, \ldots, t_{i n}\right)\right)
$$

since $\left(t_{i 1}, \ldots, t_{i n}\right)=\left(t_{j 1}, \ldots, t_{j n}\right)$. That is,

$$
\Phi_{U_{i}} \times \Phi_{U_{j}}\left(\left(t_{i 1}, \ldots, t_{i n}\right),\left(t_{j 1}, \ldots t_{j n}\right)\right)
$$

is the retraction of the same point in $X_{i} \vee X_{j}$ to both $X_{i}$ and $X_{j}$. It follows that $T_{n}^{\prime \prime}(\Phi)(t)=e$.

There is another collection of subspaces of $I^{n(n-1)}$ which will be mapped by $T_{n}^{\prime \prime}(\Phi)$ to the basepoint in $\bigwedge_{i=1}^{n} X_{i}$. These are the points in $I^{n(n-1)}$ for which one of the coordinates is 1 . We have the following result.

Lemma 4.6. Let $Z=\left\{t \in I^{n(n-1)} \mid t_{i j}=1\right.$ for some $\left.1 \leq i, j \leq n\right\}$. If $t \in Z$, and $\Phi \in \chi_{n} I\left(X_{1}, X_{2}, \ldots, X_{n}\right)$, then $T_{n}^{\prime \prime}(\Phi)(t)$ is the basepoint in $\bigwedge_{i=1}^{n} X_{i}$.

Proof. The proof follows immediately from property (b) of Definition 4.3.

Now we may define $\Delta_{n}, M_{n}$, and $T_{n}$.

Definition 4.7. Let $\Delta_{n}=I^{n(n-1)} /\left\{Z \cup \bigcup_{i<j} W_{i j}\right\}$, the quotient of $I^{n(n-1)}$ over $\left\{Z \cup \bigcup_{i<j} W_{i j}\right\}$.

Definition 4.8. $M_{n}$ is a functor of $n$ spaces defined as

$$
M_{n}\left(X_{1}, X_{2}, \ldots, X_{n}\right)=\operatorname{Map}_{*}\left(\Delta_{n}, X_{1} \wedge X_{2} \wedge \cdots \wedge X_{n}\right) .
$$

Definition 4.9. Let $T_{n}: \chi_{n} I\left(X_{1}, X_{2}, \ldots, X_{n}\right) \rightarrow M_{n}\left(X_{1}, X_{2}, \ldots, X_{n}\right)$ be the map $T_{n}^{\prime \prime}$ defined above. For $\Phi \in \chi_{n} I\left(X_{1}, X_{2}, \ldots, X_{n}\right), T_{n}^{\prime \prime}(\Phi)$ is a well-defined map by the previous lemmas.

We conclude this section by noting that $T_{n}$ is a $\Sigma_{n}$-equivariant transformation.

Theorem 2.2a. The transformation $T_{n}$ is $\Sigma_{n}$-equivariant, that is, it preserves the $\Sigma_{n}$-symmetry of $\chi_{n} I$ and $M_{n}$ which permutes the spaces $X_{1}, X_{2}, \ldots, X_{n}$ and images of the $(n-1)$-cubes in $\Delta_{n}$.

The proof follows from the definitions involved and is left to the reader.

\section{THE HOMOTOPY TYPE OF THE DERIVATIVES}

In this section, we determine the homotopy type of $\Omega \chi_{n}(\Sigma)\left(X_{1}, \ldots, X_{n}\right)$ and $\Delta_{n}$, and consequently, the homotopy type of the derivatives.

In proving Theorem $2.2 \mathrm{~b}$, we will be working with the cross effect of the functor $\Omega \Sigma$. Note that

$$
\chi_{n}(\Omega \Sigma)\left(X_{1}, X_{2}, \ldots, X_{n}\right) \simeq \chi_{n} \Omega\left(\Sigma X_{1} \wedge \cdots \wedge \Sigma X_{n}\right) \simeq \Omega \chi_{n} I\left(X_{1}, X_{2}, \ldots, X_{n}\right) .
$$

The latter equivalence comes from the fact that the loop space functor is a homotopy fiber and as such commutes with the homotopy fibers of $\chi_{n}$.

It suffices to study the cross effect of $\Omega \Sigma$ because the multilinearizations of $\chi_{n} I$ and $\chi_{n}(\Omega \Sigma)$ are equivalent, by Proposition 4.1. The convenience of this approach is that the Hilton-Milnor theorem will allow us to determine the homotopy type of $\chi_{n}(\Omega \Sigma)\left(X_{1}, X_{2}, \ldots, X_{n}\right)$ in the range we need. Specifically, we have the following. 
Proposition 5.1. If $X_{1}, \ldots, X_{n}$ are $k$-connected spaces, then

$$
\pi_{m}\left(\prod_{j=1}^{(n-1) !} \bigwedge_{i=1}^{n} X_{i}\right) \cong \pi_{m}\left(\chi_{n}(\Omega \Sigma)\left(X_{1}, \ldots, X_{n}\right)\right)
$$

for $0 \leq m \leq(n+1)(k+1)-1$.

To prove Proposition 5.1, we recall the Hilton-Milnor theorem and the definitions needed to state it. The subsequent discussion follows that of Whitehead ([Wh], pp. 511-517). Hall ([Ha]) has shown that the free Lie algebra generated by the elements $x_{1}, x_{2}, \ldots, x_{n}$ has an additive basis consisting of certain "basic products". The basic products are defined in terms of three numbers assigned to each product: the serial number, $s$, the rank, $r$, and the weight, $w$. The basic products are defined inductively by weight. The basic products of weight 1 are $x_{1}, x_{2}, \ldots, x_{n}$ with $s\left(x_{i}\right)=i$ and $r\left(x_{i}\right)=0$ for $i=1,2, \ldots, n$. Suppose that the basic products of weight $\leq m-1$ have been defined, along with their serial numbers and rank, in such a way that if $w(u)<w(v)$, then $s(u)<s(v)$. Then, the basic products of weight $m$ are all products, $u v$, where $u$ and $v$ are basic products satisfying the following conditions:

(i) $w(u)+w(v)=m$,

(ii) $s(v)<s(u)$,

(iii) $r(u) \leq s(v)$.

For such a product, $r(u v)=s(v)$. If $k$ is the largest serial number assigned to the products of weight less than or equal to $m-1$, then the products of weight $m$ can be assigned serial numbers in any order beginning with $k+1$.

A formula due to Witt [Wi] tells us that the number of basic products involving $x_{j}$ exactly $k_{j}$ times is

$$
\frac{1}{k} \sum_{d \mid k_{0}} \mu(d) \frac{(k / d) !}{\left(k_{1} / d\right) ! \cdots\left(k_{n} / d\right) !},
$$

where $k=k_{1}+\cdots+k_{n}, \mu$ is the Möbius function, and $k_{0}$ is the greatest common divisor of $k_{1}, \ldots, k_{n}$.

Given spaces $X_{1}, X_{2}, \ldots, X_{n}$, one can define the basic products of these spaces by using the smash product. Let $w_{k}\left(X_{1}, \ldots, X_{n}\right)$ denote the $k$ th basic product of the spaces $X_{1}, X_{2}, \ldots, X_{n}$. For a space $X$, recall that the space $J(X)$ is the reduced product space defined by James ([Ja]). One can define a map $h: \prod_{i=1}^{\infty} J w_{i}\left(X_{1}, \ldots, X_{n}\right) \rightarrow J\left(X_{1} \vee \cdots \vee X_{n}\right)$ (see [Wh] for details). With this we can state the Hilton-Milnor theorem ([Hi], [M]).

Theorem 5.3 (Hilton-Milnor). The map

$$
h: \prod_{i=1}^{\infty} J\left(w_{i}\left(X_{1}, \ldots, X_{n}\right)\right) \rightarrow J\left(X_{1} \vee \cdots \vee X_{n}\right)
$$

is a homotopy equivalence.

Recall that for any space $X$, there is a weak homotopy equivalence, $J(X) \rightarrow$ $\Omega \Sigma X$. This weak equivalence leads to the corollary of the Hilton-Milnor theorem stated below. 
Corollary 5.4. The spaces $\prod_{i=1}^{\infty} \Omega \Sigma\left(w_{i}\left(X_{1}, \ldots, X_{n}\right)\right)$ and $\Omega \Sigma\left(X_{1} \vee \cdots \vee X_{n}\right)$ have the same homotopy type.

Proof of Proposition 5.1. Applying Corollary 5.4 we see that

$$
\chi_{n}(\Omega \Sigma)\left(X_{1}, \ldots, X_{n}\right) \simeq \prod \Omega \Sigma\left(w_{i}\left(X_{1}, \ldots, X_{n}\right)\right) .
$$

The product on the right is taken over all basic products $w_{i}\left(X_{1}, \ldots, X_{n}\right)$ which include each $X_{j}$ at least once. Clearly, the first basic products to satisfy this condition are those involving each space exactly once. By formula (5.2) the number of such products is $(n-1)$ !. Note also that if $w_{i}\left(X_{1}, \ldots, X_{n}\right)$ is a basic product of weight $t$ then $\Omega \Sigma w_{i}\left(X_{1}, \ldots, X_{n}\right)$ is $(t(k+1)-1)$-connected. Since $\prod_{j=1}^{(n-1) !} \Omega \Sigma\left(\bigwedge_{i=1}^{n} X_{i}\right)$ and $\chi_{n}(\Omega \Sigma)\left(X_{1}, \ldots, X_{n}\right)$ differ by basic products of weight $n+1$ and greater, it follows that

$$
\pi_{m}\left(\prod_{j=1}^{(n-1) !} \Omega \Sigma\left(\bigwedge_{i=1}^{n} X_{i}\right)\right) \cong \pi_{m}\left(\chi_{n}(\Omega \Sigma)\left(X_{1}, \ldots, X_{n}\right)\right)
$$

for $0 \leq m \leq((n+1)(k+1)-1)$. Consequently,

$$
\pi_{m}\left(\prod_{j=1}^{(n-1) !}\left(\bigwedge_{i=1}^{n} X_{i}\right)\right) \cong \pi_{m}\left(\chi_{n}(\Omega \Sigma)\left(X_{1}, \ldots, X_{n}\right)\right)
$$

in the same range.

It should be noted here that, although the Hilton-Milnor theorem gives us the homotopy type of the $n$th cross effect in the range we need, the map involved does not respect the $\Sigma_{n}$-symmetry of $\chi_{n}(\Omega \Sigma)\left(X_{1}, X_{2}, \ldots, X_{n}\right)$. Hence, we have no way of recovering the $\Sigma_{n}$-action after the multilinearization of $\chi_{n}(\Omega \Sigma)\left(X_{1}, X_{2}, \ldots, X_{n}\right)$ from the information yielded by Hilton-Milnor. This is what first motivated the construction of the functor $M_{n}$.

We now turn our attention to the complex $\Delta_{n}$. For the purpose of proving the connectivity of $T_{n}$, we will use an equivalent subcomplex of $\Delta_{n}$, which we call $\widetilde{\Delta}_{n}$.

Definition 5.5. $\widetilde{\Delta}_{n}$ is the subspace of $\Delta_{n}$ consisting of all points $\left[t_{i j}\right]_{1 \leq i, j \leq n}$ in $\Delta_{n}$ such that $t_{i j}=0$ when $j \neq 1$.

The homotopy type of $\widetilde{\Delta}_{n}$ is easily determined.

Proposition 5.6. $\widetilde{\Delta}_{n}$ is homotopy equivalent to $\bigvee_{(n-1) !} S^{n-1}$.

To prove Proposition 5.6 we will construct maps labelled by elements of the following set, $G_{n}$. We will refer to this set throughout the remainder of this paper.

Definition 5.7. $G_{n}$ is the set of all bijections, $g: \underline{n} \rightarrow \underline{n}$, such that $g(1)=1$. $l \in G_{n}$ will always denote the identity.

Proof of 5.6. Let $g \in G_{n}$. Define $h_{g}: I^{n-1} \rightarrow I^{n(n-1)}$ as follows.

$$
h_{g}:\left(s_{1}, s_{2}, \ldots, s_{n-1}\right) \longmapsto\left(\begin{array}{cccc}
0 & 0 & \ldots & 0 \\
t_{21} & 0 & \ldots & 0 \\
t_{31} & 0 & \ldots & 0 \\
\vdots & & & \vdots \\
t_{n 1} & 0 & \ldots & 0
\end{array}\right)
$$


where

$$
t_{j 1}\left(s_{1}, s_{2}, \ldots, s_{n-1}\right)=\max \left\{s_{l} \mid l<g^{-1}(j)\right\} .
$$

Specifically, the image of $h_{g}$ in $I^{n(n-1)}$ is the $(n-1)$-cell in which $t_{g(2) 1}<$ $t_{g(3) 1}<\cdots<t_{g(n) 1}$. As a map of $I^{n-1}$ into $I^{n(n-1)}, h_{g}$ maps the boundary of $I^{n-1}$ to the subspaces $W_{1 g(2)}, W_{g(2) g(3)}, \ldots, W_{g(n-1) g(n)}$, and $Z$ of $I^{n(n-1)}$. That is, we have

$$
\left.h_{g}\right|_{s_{i}=0}: I^{n-1} \longrightarrow W_{g(i) g(i+1)}
$$

and

$$
\left.h_{g}\right|_{s_{j}=1}: I^{n-1} \longrightarrow Z
$$

Furthermore, if $s_{i} \geq s_{j}$ for some $i<j$ then $h_{g}$ takes the point to $W_{g(j) g(j+1)}$. To see these facts, note that in $\widetilde{\Delta}_{n}, W_{k l}$ has the form

$$
W_{k l}=\left\{t \in I^{n(n-1)} \mid t_{i j}=0 \text { for } j \neq 1 \text {, and } t_{k 1}=t_{l 1}\right\} .
$$

If $s_{i}=0$, then

$$
\begin{aligned}
t_{g(i+1) 1}\left(s_{1}, \ldots, s_{n-1}\right) & =\max \left\{s_{l} \mid l<i+1\right\}=\max \left\{s_{l} \mid l<i\right\} \\
& =t_{g(i) 1}\left(s_{1}, \ldots, s_{n-1}\right) .
\end{aligned}
$$

Thus, $h_{g}\left(s_{1}, \ldots, s_{n-1}\right) \in W_{g(i) g(i+1)}$ when $s_{i}=0$.

Furthermore, if $s_{j}=1$, then

$$
t_{g(j+1) 1}=\max \left\{s_{l} \mid l<j+1\right\}=1 .
$$

So, $h_{g}\left(s_{1}, \ldots, s_{n-1}\right) \in Z$ when any of the $s_{j}$ 's are 1 . Let $L$ be the set of all the points just identified above, whose images are in $\left(\bigcup_{i<j} W_{i j}\right) \cup Z$. That is, $L=\left\{\left(s_{1}, \ldots, s_{n-1}\right) \in I^{n-1} \mid s_{i}=0\right.$ or 1 for some $i$ or $s_{i} \geq s_{j}$ for some $\left.i<j\right\}$. From the above we can see that if we compose with the quotient map $q$ : $I^{n(n-1)} \rightarrow \Delta_{n}$, then $\lambda_{g}=q \circ h_{g}$ maps $I^{n-1} / L$ in a one-to-one manner to $\widetilde{\Delta}_{n}$. Note also that $I^{n-1} / L$ is homotopy equivalent to $S^{n-1}$ since $L$ is equivalent to $\partial I^{n-1}$. Hence, the map

$$
\bigvee_{g \in G_{n}} \lambda_{g}: \bigvee_{g \in G_{n}} S^{n-1} \rightarrow \widetilde{\Delta}_{n}
$$

is a homotopy equivalence.

As claimed we also have the following proposition and its corollaries.

Proposition 5.8. $\Delta_{n}$ and $\widetilde{\Delta}_{n}$ are weakly homotopy equivalent.

Corollary 5.9. $\Delta_{n}$ is equivalent to $\bigvee_{(n-1) !} S^{(n-1)}$.

\section{Corollary 5.10.}

$$
\operatorname{Map}_{*}\left(\Sigma \Delta_{n}, \Sigma X_{1} \wedge \Sigma X_{2} \wedge \cdots \wedge \Sigma X_{n}\right) \simeq \operatorname{Map}_{*}\left(\Sigma \tilde{\Delta}_{n}, \Sigma X_{1} \wedge \Sigma X_{2} \wedge \cdots \wedge \Sigma X_{n}\right) .
$$

And, both of these spaces are equivalent to $\prod_{(n-1) !} \Omega^{n} \Sigma^{n}\left(X_{1} \wedge X_{2} \wedge \cdots \wedge X_{n}\right)$.

The proof of Proposition 5.8 is broken up into several lemmas. Basically, we show that the space $\left(\bigcup_{i<j} W_{i j}\right) \cup Z$ is equivalent to its intersection with $\widetilde{\Delta}_{n}$. We will rely on a nice relationship between graphs and the $W_{i j}$ 's in order to better understand the structure of $\left(\bigcup_{i<j} W_{i j}\right) \cup Z$. 
Lemma 5.11. The intersection, $\bigcap_{k=1}^{s} W_{i_{k} j_{k}}$ in $I^{n(n-1)}$, for some collection of pairs, $\left\{\left(i_{k}, j_{k}\right)\right\}_{k=1}^{s}$, consists of one point, the zero matrix [0], if and only if the graph, $\Upsilon$, consisting of vertices $1,2, \ldots, n$ and edges $i_{k}-j_{k}$ for each pair $\left(i_{k}, j_{k}\right) \in\left\{\left(i_{k}, j_{k}\right)\right\}_{k=1}^{s}$ is connected.

Proof. We will first consider the case where, for each $1 \leq l \leq s$, the graphs consisting of vertices $i_{k}, j_{k}$, and edges $i_{k}-j_{k}$ for each pair $\left(i_{k}, j_{k}\right) \in\left\{\left(i_{k}, j_{k}\right)\right\}_{k=1}^{l}$ is connected, and for each $1 \leq q \leq s, i_{q} \in\left\{i_{k}, j_{k}\right\}_{k=1}^{q-1}$, and $j_{q} \notin\left\{i_{k}, j_{k}\right\}_{k=1}^{q-1}$. That is, adding the edge $i_{k}-j_{k}$ connects one new point, $j_{k}$, to the graph built out of the previous $k-1$ pairs. In this case we claim that for any point in the corresponding intersection, $\bigcap_{k=1}^{s} W_{i_{k} j_{k}}$, at least $n+s(s+1)$ of the coordinates must be equal to 0 . To show that this is the case, we proceed inductively. If we have the graph $i-j$, then the claim holds since $t_{11}, t_{22}, \ldots, t_{n n}, t_{i j}$, and $t_{j i}$ must equal 0 in $W_{i j}$. Adding the edge $i_{s}-j_{s}$ to the graph corresponding to the pairs $\left\{\left(i_{k}, j_{k}\right)\right\}_{k=1}^{s-1}$ means that for a point in $\bigcap_{k=1}^{s} W_{i_{k} j_{k}}, 2 s$ more coordinates must be zero in addition to the $n+(s-1) s$ coordinates which must be zero for points in $\bigcap_{k=1}^{s-1} W_{i_{k} j_{k}}$. Specifically, these new coordinates are $\left\{t_{s_{s} i_{k}}\right\}_{k=1}^{s}$ and $\left\{t_{i_{k} j_{s}}\right\}_{k=1}^{s}$. Thus, $n+2 s+s(s-1)=n+s(s+1)$ coordinates of a point in $\bigcap_{k=1}^{s} W_{i_{k} j_{k}}$ must be zero.

In general, we consider the connected components of $\Upsilon$ and label them $\Upsilon_{1}, \Upsilon_{2}, \ldots, \Upsilon_{a}$. Furthermore, let $W_{\Upsilon_{n}}$ denote the intersection of the $W_{i j}$ 's corresponding to the edges in $\Upsilon_{\alpha}$. Without loss of generality, we may assume that each $\Upsilon_{\alpha}$ contains a minimal number of edges, i.e., if $\Upsilon_{\alpha}$ has $r_{\alpha}$ vertices, then it has $\left(r_{\alpha}-1\right)$ edges. (Adding an extra edge to $\Upsilon_{\alpha}$ imposes no new conditions on the coordinates of a point in $W_{\Upsilon_{x}}$.) Then, by the above, a point in $W_{\Upsilon_{\alpha}}$ must have $n+r_{\alpha}\left(r_{\alpha}-1\right)$ coordinates equal to 0 . Furthermore, a point in $\bigcap_{k=1}^{s} W_{i_{k} j_{k}}=\bigcap_{\alpha=1}^{a} W_{\Upsilon_{\alpha}}$ must have at least $n+\sum_{\alpha=1}^{a} r_{\alpha}\left(r_{\alpha}-1\right)$ coordinates equal to 0 and can have as many as $\sum_{1 \leq \alpha<\beta \leq a} 2 r_{\alpha} r_{\beta}$ non-zero coordinates. Thus, if $\Upsilon$ has only one connected component, then $n+\sum_{\alpha=1}^{a} r_{\alpha}\left(r_{\alpha}-1\right)=n^{2}$ and $\bigcap_{k=1}^{s} W_{i_{k} j_{k}}$ contains only the zero matrix. If $\Upsilon$ has more than one connected component, then $\sum_{1 \leq \alpha<\beta \leq a} 2 r_{\alpha} r_{\beta}>0$ so $\bigcap_{k=1}^{s} W_{i_{k} j_{k}}$ contains points with nonzero coordinates. With this we have proved the lemma.

Lemma 5.12. The intersection, $\bigcap_{k=1}^{s} \widetilde{W}_{i_{k} j_{k}}$, in $\widetilde{I}^{n(n-1)}$ for some collection of pairs, $\left\{\left(i_{k}, j_{k}\right)\right\}_{k=1}^{s}$, is the zero matrix if and only if the graph, $\Upsilon$, consisting of points $1,2, \ldots, n$ and edges $i_{k}-j_{k}$ for each $\left(i_{k}, j_{k}\right) \in\left\{\left(i_{k}, j_{k}\right)\right\}_{k=1}^{s}$ is connected.

Proof. Let $t=\left[t_{i j}\right]$ be a point in $\bigcap_{k=1}^{s} \widetilde{W}_{i_{k} j_{k}}$. It is easy to see that $t_{i_{k} 1}=0$ if and only if the point $i_{k}$ is part of the connected component of $\Upsilon$ containing 1 . Hence, all the $t_{i 1}$ 's are equal to zero if and only if $\Upsilon$ is connected, otherwise $\bigcap_{k=1}^{n} \widetilde{W}_{i_{k} j_{k}}$ will contain points with nonzero coordinates.

Lemma 5.13. If the graph, $\Upsilon$, associated to the collection of pairs $\left\{\left(i_{k}, j_{k}\right)\right\}_{k=1}^{s}$ is not connected then $\bigcap_{k=1}^{s} W_{i_{k} j_{k}}$ and $\bigcap_{k=1}^{s} \widetilde{W}_{i_{k} j_{k}}$ are contractible to points in $Z$ and $\tilde{Z}$ respectively.

Proof. By Lemma 5.12, if $\Upsilon$ is not connected then for a point in $W_{\Upsilon}$, there is at least one coordinate which does not have to be 0 . Let $t_{1}, \ldots, t_{m}$ be those coordinates which do not have to be 0 . Let $H: \bigcap_{k=1}^{s} W_{i_{k} j_{k}} \times I \rightarrow \bigcap_{k=1}^{s} W_{i_{k} j_{k}}$ be 
the homotopy which takes each $t_{l} \in\left\{t_{1}, \ldots, t_{m}\right\}$ and $t \in I$, to $t\left(1-t_{l}\right)+t_{l}$. The argument is similar for $\bigcap_{k=1}^{s} \widetilde{W}_{i_{k} j_{k}}$.

As easy consequences of these lemmas, we have the following corollaries.

Corollary 5.14. Let $\Upsilon$ be the graph associated to the collection of pairs $\left\{\left(i_{k}, j_{k}\right)\right\}_{k=1}^{s}, W_{\Upsilon}$ be the corresponding intersection $\bigcap_{k=1}^{s} W_{i_{k} j_{k}}$, and $\widetilde{W}_{\Upsilon}$ be the corresponding intersection $\bigcap_{k=1}^{s} \widetilde{W}_{i_{k} j_{k}}$. If $\Upsilon$ is connected then $W_{\Upsilon} \cap Z$ and $\widetilde{W}_{\Upsilon} \cap \tilde{Z}$ are empty.

Corollary 5.15. For any collection of pairs $\left\{\left(i_{k}, j_{k}\right)\right\}_{k=1}^{s}$, the inclusions $\bigcap_{k=1}^{s} \widetilde{W}_{i_{k} j_{k}} \hookrightarrow \bigcap_{k=1}^{s} W_{i_{k} j_{k}}$ and $\left(\bigcap_{k=1}^{s} \widetilde{W}_{i_{k} j_{k}}\right) \cap \tilde{Z} \hookrightarrow\left(\bigcap_{k=1}^{s} W_{i_{k} j_{k}}\right) \cap Z$ are homotopy equivalences.

The corollaries allow us to prove the next proposition.

Proposition 5.16. The inclusion $\widetilde{W} \cup \widetilde{Z} \hookrightarrow W \cup Z$ is a weak homotopy equivalence.

For the proof of this proposition we need the following version of the gluing lemma found in [Wa].

Lemma 5.17. In the commutative diagram

$$
\begin{gathered}
X_{1} \leftarrow X_{0} \rightarrow X_{2} \\
\downarrow \\
Y_{1} \\
\downarrow
\end{gathered}
$$

let the two left horizontal maps be cofibrations and suppose that all the vertical maps are weak equivalences. Then the map of pushouts $X_{1} \cup_{X_{0}} X_{2} \rightarrow Y_{1} \cup_{Y_{0}} Y_{2}$ is also a weak equivalence.

Proof of 5.16. With repeated applications of the gluing lemma to diagrams of the form

and

$$
\begin{aligned}
& \bigcap_{k=1, k \neq m}^{s} \widetilde{W}_{i_{k} j_{k}} \leftarrow \bigcap_{k=1}^{s} \widetilde{W}_{i_{k} j_{k}} \rightarrow \bigcap_{k=1, k \neq l}^{s} \widetilde{W}_{i_{k} j_{k}} \\
& \bigcap_{k=1, k \neq m}^{s} W_{i_{k} j_{k}} \leftarrow \bigcap_{k=1}^{s} W_{i_{k} j_{k}} \rightarrow \bigcap_{k=1, k \neq l}^{s} W_{i_{k} j_{k}}
\end{aligned}
$$

$$
\begin{array}{ccccc}
\tilde{Z} & \leftarrow & \widetilde{W}_{\Upsilon} \cap \tilde{Z} & \rightarrow & \widetilde{W}_{\Upsilon} \\
\downarrow & & \downarrow & & \downarrow \\
Z & \leftarrow & W_{\Upsilon} & \rightarrow & W_{\Upsilon}
\end{array}
$$

we are able to show that $\widetilde{W} \cup \widetilde{Z} \hookrightarrow W \cup Z$ is a homotopy equivalence.

Since $\widetilde{I}^{(n-1)} \hookrightarrow I^{n(n-1)}$ is also an equivalence, the quotients $\widetilde{\Delta}_{n}$ and $\Delta_{n}$ are equivalent and the proof of Proposition 5.8 is complete.

The proof of the second statement of Corollary 5.10 is as follows.

Proof of 5.10. As a consequence of 5.6, each of the spaces is equivalent to $\operatorname{Map}_{*}\left(\Sigma\left(\bigvee_{(n-1) !} S^{(n-1)}\right), \Sigma X_{1} \wedge \cdots \wedge \Sigma X_{n}\right)$. Furthermore,

$$
\operatorname{Map}_{*}\left(\Sigma\left(\bigvee_{(n-1) !} S^{n-1}\right), \Sigma X_{1} \wedge \cdots \wedge \Sigma X_{n}\right)
$$

is equivalent to $\prod_{(n-1) !} \operatorname{Map}_{*}\left(S^{n}, S^{n} \wedge\left(X_{1} \wedge \cdots \wedge X_{n}\right)\right)$, and this is equivalent to $\prod_{(n-1) !} \Omega^{n} \Sigma^{n}\left(X_{1} \wedge X_{2} \wedge \cdots \wedge X_{n}\right)$. 


\section{THE CONNECTIVITY OF $\Omega T_{n}$}

We have shown that for $m \leq(n+1)(k+1)-1, \pi_{m}\left(\prod_{(n-1) !} \bigwedge_{i=1}^{n} X_{i}\right)$ is isomorphic to $\pi_{m}\left(\chi_{n}(\Omega \Sigma)\left(X_{1}, X_{2}, \ldots, X_{n}\right)\right)$ when $X_{1}, X_{2}, \ldots, X_{n}$ are $k$-connected spaces. From Corollary 5.10 it follows that $\pi_{m}\left(\chi_{n}(\Omega \Sigma)\left(X_{1}, X_{2}, \ldots, X_{n}\right)\right)$ and $\pi_{m}\left(\operatorname{Map}_{*}\left(\Sigma \Delta_{n}, X_{1} \wedge X_{2} \wedge \cdots \wedge X_{n}\right)\right)$ are isomorphic for $m \leq(n+1)(k+1)-1$. Now it remains to show that $\left(\Omega T_{n}\right)_{*}$ induces this isomorphism. To do so, we will set up the commutative diagram below

$$
\begin{array}{ccc}
\widetilde{H}_{*}\left(\bigvee_{g \in G_{n}}\left(\prod_{i=1}^{n} X_{i}\right)\right) \stackrel{\left(\left(\Omega T_{n}\right) \circ D\right)_{*}}{\longrightarrow} & \widetilde{H}_{*}\left(\operatorname{Map}_{*}\left(\Sigma \widetilde{\Delta}_{n}, \Sigma X_{1} \wedge \cdots \wedge \Sigma X_{n}\right)\right) \\
\downarrow & & \widetilde{H}_{*}\left(\prod_{i=1}^{(n-1) !}\left(\Omega^{n} \Sigma^{n}\left(X_{1} \wedge \cdots \wedge X_{n}\right)\right)\right) \\
& & \\
\widetilde{H}_{*}\left(\bigvee_{g \in G_{n}}\left(\bigwedge_{i=1}^{n} X_{i}\right)\right) & \longrightarrow & \bigoplus_{g \in G_{n}} \widetilde{H}_{*}\left(\Omega^{n} \Sigma^{n}\left(X_{1} \wedge \cdots \wedge X_{n}\right)\right)
\end{array}
$$

The first step will be to define the maps in the diagram and compute the degree of those maps.

For each $g \in G_{n}$ we will construct a map $C_{g}$ that maps $\prod_{i=1}^{n} X_{i}$ to $\chi_{n}(\Omega \Sigma)\left(X_{1}, \ldots, X_{n}\right)$. Like the map used to prove the Hilton-Milnor theorem (see [Hi], [M]), $C_{g}$ will be defined via commutators. In order to construct $C_{g}$, we will need a few basic maps. These maps, $A, B, C$, and $P$, will provide standard homotopies from commutator loops to constant loops, and will allow us to build elements of $\chi_{n}(\Omega \Sigma)$ from elements of $\chi_{k}(\Omega \Sigma)$ for $k<n$.

Definition 6.2. $C: \Omega X \times \Omega Y \rightarrow \Omega(X \vee Y)$ is the commutator. That is, for $\alpha \in \Omega X$, and $\beta \in \Omega Y, C(\alpha, \beta)$ is the commutator loop $\alpha \beta \alpha^{-1} \beta^{-1}$.

Definition 6.3. $A: I^{2} \rightarrow I$ is defined by

$$
A(s, t)= \begin{cases}\frac{4 t}{s+1}, & \text { for } 0 \leq s \leq \frac{1}{2} \text { and } 0 \leq t \leq\left(\frac{1}{4}+\frac{1}{4} s\right) \\ 1, & \text { for } 0 \leq s \leq \frac{1}{2} \text { and }\left(\frac{1}{4}+\frac{1}{4} s\right) \leq t \leq\left(\frac{1}{2}-\frac{1}{4} s\right) \\ \frac{3-4 t}{s+1}, & \text { for } 0 \leq s \leq \frac{1}{2} \text { and }\left(\frac{1}{2}-\frac{1}{4} s\right) \leq t \leq \frac{3}{4} \\ 0, & \text { for } 0 \leq s \leq \frac{1}{2} \text { and } \frac{3}{4} \leq t \leq 1 \\ 0, & \text { for } \frac{1}{2} \leq s \leq 1 \text { and } 0 \leq t \leq \frac{3}{4}\left(s-\frac{1}{2}\right) \\ \frac{8 t-6 s+3}{3}, & \text { for } \frac{1}{2} \leq s \leq 1 \text { and } \frac{3}{4}\left(s-\frac{1}{2}\right) \leq t \leq \frac{3}{8} \\ \frac{-8 t-6 s+9}{3}, & \text { for } \frac{1}{2} \leq s \leq 1 \text { and } \frac{3}{8} \leq t \leq \frac{9}{8}-\frac{3}{4} s \\ 0, & \text { for } \frac{1}{2} \leq s \leq 1 \text { and } \frac{9}{8}-\frac{3}{4} s \leq t \leq 1 .\end{cases}
$$

Note that, if $e$ is the constant loop at the basepoint in $X$, and $\alpha \in \Omega X$, then $\alpha(A(s, t))$ is a homotopy from the commutator loop $C(\alpha, e)$ to $e$. See Figure 1 . 


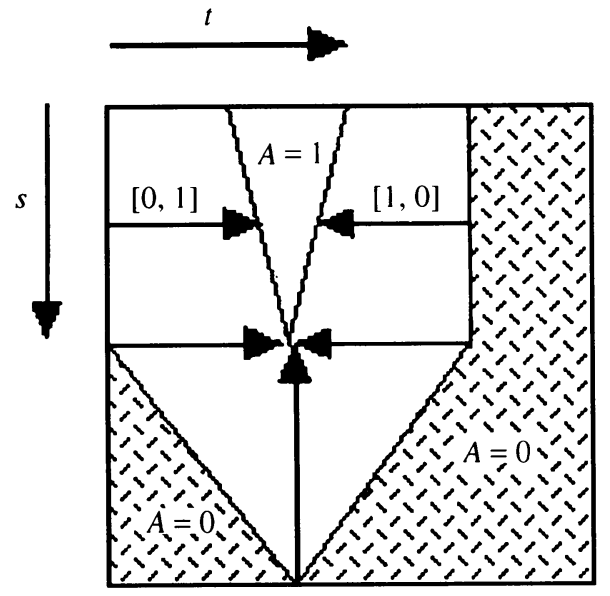

FIGURE 1

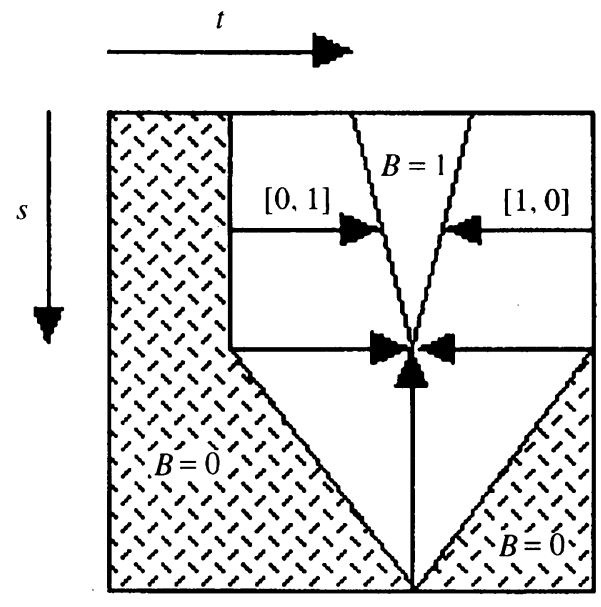

FIGURE 2

Definition 6.4. $B: I^{2} \rightarrow I$ is defined by

$$
B(s, t)= \begin{cases}0, & \text { for } 0 \leq s \leq \frac{1}{2} \text { and } 0 \leq t \leq \frac{1}{4} \\ \frac{4 t-1}{s+1}, & \text { for } 0 \leq s \leq \frac{1}{2} \text { and } \frac{1}{4} \leq t \leq \frac{1}{2}+\frac{1}{4} s \\ 1, & \text { for } 0 \leq s \leq \frac{1}{2} \text { and } \frac{1}{2}+\frac{1}{4} s \leq t \leq \frac{3}{4}-\frac{1}{4} s \\ \frac{4-4 t}{s+1}, & \text { for } 0 \leq s \leq \frac{1}{2} \text { and } \frac{3}{4}-\frac{1}{4} s \leq t \leq 1 \\ 0, & \text { for } \frac{1}{2} \leq s \leq 1 \text { and } 0 \leq t \leq \frac{3}{4} s-\frac{1}{8} \\ \frac{8 t-6 s+1}{3}, & \text { for } \frac{1}{2} \leq s \leq 1 \text { and } \frac{3}{4} s-\frac{1}{8} \leq t \leq \frac{5}{8} \\ \frac{-8 t-6 s+11}{3}, & \text { for } \frac{1}{2} \leq s \leq 1 \text { and } \frac{5}{8} \leq t \leq \frac{11}{8}-\frac{3}{4} s \\ 0, & \text { for } \frac{1}{2} \leq s \leq 1 \text { and } \frac{11}{8}-\frac{3}{4} s \leq t \leq 1 .\end{cases}
$$

Note that, if $e$ is the constant loop at the basepoint in $X$, and $\beta \in \Omega X$, then $\beta(B(s, t))$ is a homotopy from the commutator loop $C(e, \beta)$ to $e$. See Figure 2.

Finally, we define a map

$$
P: \chi_{n-1} \Omega\left(X_{1}, X_{2}, \ldots, X_{n-1}\right) \times \chi_{1} \Omega\left(X_{n}\right) \rightarrow \chi_{n} \Omega\left(X_{1}, X_{2}, \ldots, X_{n}\right) .
$$

Essentially, $P$ builds an element of $\chi_{n} \Omega\left(X_{1}, X_{2}, \ldots, X_{n}\right)$ out of the commutator of a pair of elements from $\chi_{n-1} \Omega\left(X_{1}, X_{2}, \ldots, X_{n-1}\right)$ and $\chi_{1} \Omega\left(X_{n}\right)$. In the following $e$ will always denote the constant loop at the basepoint.

Definition 6.5. For $\Phi \in \chi_{n-1} \Omega\left(X_{1}, X_{2}, \ldots, X_{n-1}\right)$ and $\beta \in \chi_{1} \Omega\left(X_{n}\right)$, $P: \chi_{n-1} \Omega\left(X_{1}, X_{2}, \ldots, X_{n-1}\right) \times \chi_{1} \Omega\left(X_{n}\right) \rightarrow \chi_{n} \Omega\left(X_{1}, X_{2}, \ldots, X_{n}\right)$ is the element $P(\Phi, \beta)=\left\{P(\Phi, \beta)_{U}\right\}_{U \in N}$ in $\chi_{n} \Omega\left(X_{1}, X_{2}, \ldots, X_{n}\right)$. The maps $P(\Phi, \beta)_{U}: I^{U} \rightarrow \Omega\left(\bigvee_{i \notin U} X_{i}\right)$ are defined as below. $t$ will denote the loop coordinate.

(a) If $n \notin U$,

$$
P(\Phi, \beta)_{U}=\left\{\begin{array}{l}
C\left(\Phi_{U}\left(2 t_{1}, \ldots, 2 t_{n-1}\right), \beta_{\varnothing}\right)(t), \quad 0 \leq t_{1}, \ldots, t_{n-1} \leq 1 / 2, \\
\beta_{\varnothing}\left(B\left(\max \left(2 t_{1}-1, \ldots, 2 t_{n}-1\right), t\right)\right), \quad \text { otherwise }
\end{array}\right.
$$


(b) If $n \in U$,

$P(\Phi, \beta)_{U}= \begin{cases}\Phi_{U-\{n\}}\left(\left(2 t_{1}, \ldots, 2 t_{n-1}\right)\right)\left(A\left(t_{n}, t\right)\right), & 0 \leq t_{1}, \ldots, t_{n-1} \leq 1 / 2, \\ e, & \text { otherwise. }\end{cases}$

We leave it to the reader to check that $P(\Phi, \beta)$ as defined above satisfies all conditions necessary to be an element of $\chi_{n} \Omega\left(X_{1}, X_{2}, \ldots, X_{n}\right)$.

Now we may define $C_{g}$.

Definition 6.6. For $g \in G_{n}$, we define $C_{g}: \prod_{i=1}^{n} X_{i} \rightarrow \chi_{n}(\Omega \Sigma)\left(X_{1}, X_{2}, \ldots, X_{n}\right)$. The definition for any $g$ will depend on the definition of $C_{l}$ where $l$ again denotes the identity function. $C_{l}$ is defined inductively. For $n=1, C_{l}: X \rightarrow$ $\chi_{1} \Omega \Sigma X$ is the function such that

$$
\left(C_{l}(x)\right)_{\varnothing}: I^{\varnothing} \rightarrow x(t)
$$

where $x(t)$ is the loop in $\Sigma X$ which takes $t$ to $x \wedge t$. And,

$$
\left(C_{l}(x)\right)_{\{1\}}=e \text {. }
$$

For $l \in G_{n}$, where $n>1, C_{l}: \prod_{i=1}^{n} X_{i} \rightarrow \chi_{n}(\Omega \Sigma)\left(X_{1}, \ldots, X_{n}\right)$ is defined as

$$
C_{l}\left(x_{1}, \ldots, x_{n}\right)=P\left(C_{l}\left(x_{1}, x_{2}, \ldots, x_{n-1}\right), C_{l}\left(x_{n}\right)\right) \text {. }
$$

Finally, for any $g \in G_{n}, C_{g}: \prod_{i=1}^{n} X_{i} \rightarrow \chi_{n}(\Omega \Sigma)\left(X_{1}, \ldots, X_{n}\right)$ is defined as

$$
C_{g}\left(x_{1}, \ldots, x_{n}\right)=C_{l}\left(x_{g^{-1}(1)}, x_{g^{-1}(2)}, \ldots, x_{g^{-1}(n)}\right) .
$$

The maps $C_{g}$, though not explicitly the same, were inspired by the map of the Hilton-Milnor theorem. The Hilton-Milnor map takes a point in $\Omega \Sigma Y_{j}$, for some basic product $Y_{j}$, to a nested commutator of loops in $\Omega \Sigma\left(X_{1} \vee X_{2} \vee \cdots \vee X_{n}\right)$ determined by the grouping and ordering of the $X_{i}$ 's in $Y_{j}$. Essentially, $C_{g}$ takes a point $\left(x_{1}, \ldots, x_{n}\right)$ to the point in $\chi_{n} \Omega\left(\Sigma X_{1}, \Sigma X_{2}, \ldots, \Sigma X_{n}\right)$ consisting of the nested commutator loop,

$$
C\left(x_{g^{-1}(1)}, \ldots, C\left(x_{g^{-1}(n-2)}\left(C\left(x_{g^{-1}(n-1)}, x_{g^{-1}(n)}\right)\right)\right) \ldots\right)
$$

in $\Omega\left(\Sigma X_{1} \vee \Sigma X_{2} \vee \cdots \vee \Sigma X_{n}\right)$, determined by the surjection $g$, and homotopies from that loop to $e$ in $\Omega\left(\bigvee_{i \notin U} \Sigma X_{i}\right)$.

We now wish to determine the connectivity of the composition $\Omega T_{n} \circ C_{g}$, as indicated in the commutative diagram at the beginning of this section. To do so, first note that for a point $\left(x_{1}, \ldots, x_{n}\right)$ in $\prod_{i=1}^{n} X_{i},\left(\Omega T_{n}\right) \circ C_{g}\left(x_{1}, \ldots, x_{n}\right) \circ \Lambda_{h}$ ( $\Lambda_{h}$ is the suspension of the map $\lambda_{h}$ defined in the proof of Proposition 5.6) is a map from $S^{n}$ to $\Sigma^{n}\left(\bigwedge_{i=1}^{n} X_{i}\right)$. From the definition of $C_{g}$ one can see that the image of a point $s \in S^{n}$ under this map will always have the form $\Gamma_{g h}(s) \wedge\left(x_{1} \wedge \cdots \wedge x_{n}\right)$, where $\Gamma_{g h} \in \Omega^{n} \Sigma^{n}$ is a map determined by the choice of $g$ and $h$. In other words, we can make the following definition.

Definition 6.7. $\Gamma_{g h}: S^{n} \rightarrow S^{n}$ is the map which makes the diagram below commutative. ( $q$ is the quotient map.)

$$
\begin{array}{cc}
\prod_{i=1}^{n} X_{i} \stackrel{\left(\Omega T_{n}\right) \circ C_{g}}{\longrightarrow} & \operatorname{Map}_{*}\left(\Sigma \tilde{\Delta}_{n}, \Sigma X_{1} \wedge \cdots \wedge \Sigma X_{n}\right) \\
& \downarrow \Lambda_{h}^{*} \\
\bigwedge_{i=1}^{n} X_{i} \underset{x \mapsto \Gamma_{g h} \wedge x}{\longrightarrow} & \Omega^{n} \Sigma^{n}\left(\bigwedge_{i=1}^{n} X_{i}\right)
\end{array}
$$


The advantage of considering the maps $\Gamma_{g h}$ is that we can determine their degree for any choice of $g$ and $h$ in $G_{n}$.

Proposition 6.8. For $g, h \in G_{n}$, the map $\Gamma_{g h}: S^{n} \rightarrow S^{n}$ has degree one if $h=g$. Otherwise, it is null-homotopic.

The proof of Proposition 6.8 will proceed by induction on $n$. The heart of the proof will be defining a map $\rho^{*}$ that makes the following diagram commute.

$$
\begin{array}{cc}
\chi_{n-1} \Omega\left(X_{1}, \ldots, X_{n-1}\right) \times \chi_{1} \Omega\left(X_{n}\right) \stackrel{P}{\longrightarrow} \chi_{n} \Omega\left(X_{1}, X_{2}, \ldots, X_{n}\right) \\
\Omega T_{n-1} \wedge \Omega T_{1} \downarrow & \Omega T_{n} \downarrow \\
\operatorname{Map}_{*}\left(\Sigma \widetilde{\Delta}_{n-1} \wedge \Sigma \widetilde{\Delta}_{1}, \Sigma X_{1} \wedge \Sigma X_{n}\right) \stackrel{\rho^{*}}{\longrightarrow} \operatorname{Map}_{*}\left(\Sigma \widetilde{\Delta}_{n}, \Sigma X_{1} \wedge \Sigma X_{n}\right) .
\end{array}
$$

This diagram will allow us to compute the degree of the map in question by means of an equivalent map in $\operatorname{Map}_{*}\left(\Sigma \widetilde{\Delta}_{n-1} \wedge \widetilde{\Delta}_{1}, X_{1} \wedge X_{2} \wedge \cdots \wedge X_{n}\right)$, hence allowing us to set up an inductive argument to prove Proposition 6.8.

To define $\rho^{*}$, we will define a map $\rho: \Sigma \widetilde{\Delta}_{n} \rightarrow \Sigma \widetilde{\Delta}_{n-1} \wedge \widetilde{\Delta}_{1} . \rho$ necessitates the use of the complex $\widetilde{\Delta}_{n}$, since it wasn't possible to construct such a map on $\Delta_{n}$ which was well-defined. (On the other hand, $\widetilde{\Delta}_{n}$ does not suffice for our purposes because the equivalence $\widetilde{\Delta}_{n} \rightarrow \Delta_{n}$ does not preserve the $\Sigma_{n}$-symmetry.)

Definition 6.10. Let $\left(s, t_{21}, t_{31}, \ldots, t_{n 1}\right)$ be a point in $\Sigma \widetilde{\Delta}_{n}$, where $s$ denotes the suspension coordinate and $t_{21}, t_{31}, \ldots, t_{n 1}$ denote the (possibly) non-zero coordinates of a point in $\widetilde{\Delta}_{n} . \rho: \Sigma \widetilde{\Delta}_{n} \rightarrow \Sigma \widetilde{\Delta}_{n-1} \wedge \widetilde{\Sigma} \Delta_{1}$ is defined by

$$
\rho\left(s, t_{21}, t_{31}, \ldots, t_{n 1}\right)=\left(u \wedge\left(s_{21}, s_{31}, \ldots, s_{(n-1) 1}\right)\right) \wedge v
$$

where $\left(s_{21}, \ldots, s_{(n-1) 1}\right)$ are the coordinates of a point in $\tilde{\Delta}_{n-1}, u$ is the suspension coordinate of $\Sigma \widetilde{\Delta}_{n-1}$, and $v$ is a point in $\Sigma \widetilde{\Delta}_{1}$. They are defined as

$$
\begin{aligned}
s_{i 1} & =\min \left(1,2 t_{i 1}\right), \\
u & =A(0, s), \\
v & =B\left(\max \left(0,2 t_{n 1}-1\right), s\right) .
\end{aligned}
$$

The map

$$
\rho^{*}: \operatorname{Map}_{*}\left(\Sigma \tilde{\Delta}_{n-1} \wedge \Sigma \tilde{\Delta}_{1}, X_{1} \wedge X_{2} \wedge \cdots \wedge X_{n}\right) \rightarrow \operatorname{Map}_{*}\left(\Sigma \tilde{\Delta}_{n}, X_{1} \wedge X_{2} \wedge \cdots \wedge X_{n}\right)
$$

is defined as

$$
\rho^{*}: \Theta \mapsto \Theta \circ \rho \quad \text { for } \Theta \in \operatorname{Map}_{*}\left(\Sigma \tilde{\Delta}_{n-1} \wedge \Sigma \widetilde{\Delta}_{1}, X_{1} \wedge X_{2} \wedge \cdots \wedge X_{n}\right) .
$$

With these definitions, it is easily shown that (6.9) is commutative. We omit the details.

The next step is to study the maps $\rho \circ \Lambda_{g}$.

Lemma 6.11. Let $g \in G_{n}$. Consider the map $\rho \circ \Lambda_{g}: S^{n} \rightarrow \Sigma \widetilde{\Delta}_{n-1} \wedge \Sigma \widetilde{\Delta}_{1}$. When $g(n)=n$, let $f$ denote the restriction of $g$ to $\{\underline{n-1}\}$. Then,

$$
\rho \circ \Lambda_{g} \simeq \begin{cases}\Lambda_{f} \wedge \mathrm{id}_{S^{1}} & \text { if } g(n)=n, \\ e & \text { if } g(n) \neq n .\end{cases}
$$




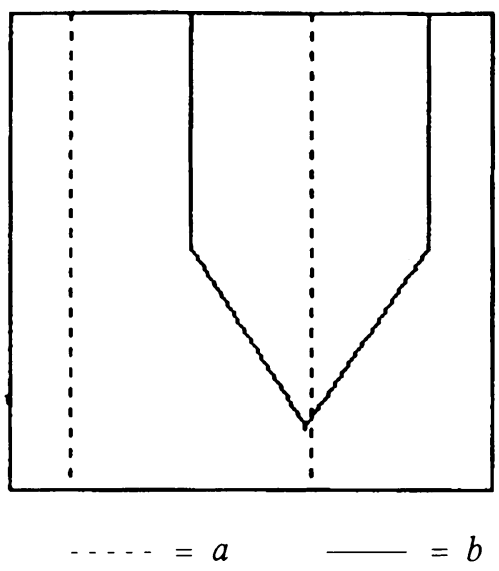

FIGURE 3

Proof. Let $t \wedge s_{1} \wedge \cdots \wedge s_{n-1}$ be a point in $S^{n}$ where $S^{n}$ is considered as the smash product of $n$ copies of $S^{1}$. If $g(n)=n$, then

$$
\begin{aligned}
\rho \circ \Lambda_{g}(t & \wedge s_{1} \wedge \\
\wedge & \cdots A(0, t) \wedge B\left(\max \left(0,2 t_{n 1}-1\right), t\right)
\end{aligned}
$$

where

$$
t_{j 1}=\max \left\{s_{l} \mid l<g^{-1}(j)\right\}
$$

If $g(n)=n$, then

$$
t_{n 1}=\max \left\{s_{1}, \ldots, s_{n-1}\right\}
$$

and $s_{n-1} \notin\left\{s_{l} \mid l<g^{-1}(j)\right\}$ for any $j \neq n$. (Thus, the image in $\widetilde{\Delta}_{n-1}$ does not depend on $s_{n-1}$.) Also note that if $\max \left(0,2 t_{n 1}-1\right)>0$ then $s_{j}>1 / 2$ for some $j$. If $j \neq n-1$, then $\min \left(1,2 t_{i 1}\right)=1$ for all $i$ with $j \leq g^{-1}(i)$, and hence $\rho \circ \Lambda_{g}\left(t \wedge s_{1} \wedge \cdots s_{n-1}\right)=e$. It follows that

$$
\rho \circ \Lambda_{g}\left(t \wedge s_{1} \wedge s_{2} \wedge \cdots \wedge s_{n-1}\right)
$$

is equal to

$$
\left(\min \left(1,2 t_{21}\right), \ldots, \min \left(1,2 t_{(n-1) 1}\right)\right) \wedge A(0, t) \wedge B\left(\max \left(0,2 s_{n-1}-1\right), t\right) .
$$

The map $\left(\min \left(1,2 t_{21}\right), \min \left(1,2 t_{31}\right), \ldots, \min \left(1,2 t_{(n-1) 1}\right)\right)$ is homotopic to $\lambda_{f}$ via the homotopy

$$
\left(\min \left(1,(2-q) t_{21}\right), \min \left(1,(2-q) t_{31}\right), \ldots, \min \left(1,(2-q) t_{(n-1) 1}\right)\right) .
$$

To see that $A(0, t) \wedge B\left(\max \left(0,2 s_{n-1}-1\right), t\right)$ is homotopic to the identity map on $S^{2}=I^{2} / \partial I^{2}$, consider a point $(a, b) \in I^{2}$ such that $a, b \neq 0,1$. The inverse image of $\{a\} \times I$ in $I^{2}$ has the form $\{a / 4,(a+3) / 4\} \times I$. The inverse image of $I \times\{b\}$ in $I^{2}$ under $B\left(\max \left(0,2 s_{n-1}-1\right), t\right)$ is a path in $I^{2}$ from $(0,(b+1) / 4)$ to $(0,(b+4) / 4)$. It is easy to see that the inverse images of $I \times\{b\}$ and $\{a\} \times I$ intersect in a single point (see Figure 3). Hence the map has degree one and is homotopic to the identity map as claimed.

If $g(n) \neq n$, then $t_{g(n) 1}=\max \left(s_{1}, s_{2}, \ldots, s_{n-1}\right)$. In order to have a nontrivial point in the image of $\rho \circ \Lambda_{g}$ we must have $\min \left(1,2 t_{g(n) 1}\right) \neq 1$, i.e., 
$s_{i}<1 / 2$ for all $i$. If this is the case then $t_{n 1}<1 / 2$, and $\max \left(0,2 t_{n 1}-1\right)=0$. Thus, if $s_{i}<1 / 2$ for all $i$, then

$$
\rho \circ \Lambda_{g}\left(t \wedge s_{1} \wedge \cdots \wedge s_{n-1}\right)
$$

is equal to

$$
\left(\min \left(1,2 t_{21}\right), \ldots, \min \left(1,2 t_{(n-1) 1}\right)\right) \wedge A(0, t) \wedge B(0, t) .
$$

But, $A(0, t) \wedge B(0, t)$ will always be the basepoint in $S^{2}$. Hence, $\rho \circ \Lambda_{g}$ is null homotopic.

With this we are now ready to prove Proposition 6.8.

Proof of Proposition 6.8. When $n=1$, we need only consider one case, $l \in G_{1}$. One can easily see that $\Gamma_{l l}$ is the identity map on $S^{1}$, and hence the proposition holds here. Furthermore, for $l \in G_{2}$,

$$
\Gamma_{u l}\left(t \wedge s_{1}\right)=A(0, t) \wedge B\left(\max \left(0,2 s_{1}-1\right), t\right)
$$

since

$$
\Omega T_{2} \circ C_{l}\left(x_{1}, x_{2}\right) \circ \Lambda_{g}\left(t \wedge s_{1}\right)=A(0, t) \wedge x_{1} \wedge\left(B\left(\max \left(0,2 s_{1}-1\right), t\right)\right) \wedge x_{2} .
$$

We saw in the proof of Lemma 6.11 that this has degree one.

For $g, h \in G_{n}$, it follows from the definitions of $C_{g}, T_{n}$ and $\Lambda_{g}$ that

$$
\left(\Omega T_{n} \circ C_{g}\left(x_{1}, x_{2}, \ldots, x_{n}\right)\right) \circ \Lambda_{h}=\left(\Omega T_{n} \circ C_{l}\left(x_{1}, x_{2}, \ldots, x_{n}\right)\right) \circ \Lambda_{g^{-1} \circ h} .
$$

Furthermore,

$$
\begin{gathered}
\left(\Omega T_{n} \circ C_{l}\left(x_{1}, \ldots, x_{n}\right)\right) \circ \Lambda_{g^{-1} \circ h}=\left(\Omega T_{n} \circ P\left(C_{l}\left(x_{1}, \ldots, x_{n}\right), C_{l}\left(x_{n}\right)\right)\right) \circ \Lambda_{g^{-1} \circ h} \\
=\Omega T_{n-1}\left(C_{l}\left(x_{1}, \ldots, x_{n-1}\right)\right) \wedge \Omega T_{1}\left(C_{l}\left(x_{n}\right)\right) \circ\left(\rho \circ \Lambda_{g^{-1} \circ h}\right) .
\end{gathered}
$$

If $g=h$, then $\rho \circ \Lambda_{g^{-1} \circ h} \simeq \Lambda_{l} \wedge \mathrm{id}_{S^{1}}$ by Lemma 6.11. So,

$$
\left(\Omega T_{n} \circ C_{g}\left(x_{1}, \ldots, x_{n}\right)\right) \circ \Lambda_{h}
$$

is equivalent to

$$
\left(\Omega T_{n-1}\left(C_{l}\left(x_{1}, \ldots, x_{n-1}\right)\right) \circ \Lambda_{l}\right) \wedge \Omega T_{1}\left(C_{l}\left(x_{n}\right)\right) \circ \mathrm{id}_{S^{1}}
$$

which is equivalent to

$$
\Gamma_{u} \wedge x_{1} \wedge \cdots \wedge x_{n-1} \wedge \Gamma_{u} \wedge x_{n}
$$

Therefore $\Gamma_{g g}$ has degree one, by induction.

If $g \neq h$, and $g^{-1} \circ h(n) \neq n$, then $\rho \circ \Lambda_{g^{-1} \circ h}$ is null homotopic, so $\Gamma_{g h}$ is as well. If $g^{-1} \circ h(n)=n$, then $\left(\Omega T_{n-1} \circ C_{l}\left(x_{1}, x_{2}, \ldots, x_{n}\right)\right) \circ \Lambda_{f}$ (where $f$ is the restriction of $g^{-1} \circ h$ to $\left.\{n-1\}\right)$ can be reduced as above to a smash product of maps, one of which will have the form $\Omega T_{n-k}\left(C_{l}\left(x_{1}, \ldots, x_{n-k}\right)\right) \circ\left(\rho \circ \Lambda_{j}\right)$ where $j(n-k) \neq n-k$. This is guaranteed by the fact that $g^{-1} \circ h \neq l$. Then Lemma 6.11 can be applied as above to determine that this component is null homotopic. Hence, we can conclude that $\Gamma_{g h}$ is null homotopic when $g \neq h$.

With this we are able to prove Theorem $2.2 \mathrm{~b}$. 
Proof of Theorem 2.2b. We will work with the commutative diagram of reduced homology groups (6.1) .

$$
\begin{array}{ccc}
\widetilde{H}_{m}\left(\bigvee_{g \in G_{n}}\left(\prod_{i=1}^{n} X_{i}\right)\right) \stackrel{\left(\left(\Omega T_{n}\right) \circ D\right)_{*}}{\longrightarrow} & \widetilde{H}_{m}\left(\operatorname{Map}_{*}\left(\Sigma \tilde{\Delta}_{n}, \Sigma X_{1} \wedge \cdots \wedge \Sigma X_{n}\right)\right) \\
& & \\
& & \widetilde{H}_{m}\left(\prod_{h \in G_{n}}\left(\Omega^{n} \Sigma^{n}\left(X_{1} \wedge \cdots \wedge X_{n}\right)\right)\right) \\
& & \\
& & \\
\widetilde{H}_{m}\left(\bigvee_{g \in G_{n}}\left(\bigwedge_{i=1}^{n} X_{i}\right)\right) & \underset{\Gamma}{ } & \bigoplus_{h \in G_{n}} \widetilde{H}_{m}\left(\Omega^{n} \Sigma^{n}\left(X_{1} \wedge \cdots \wedge X_{n}\right)\right) .
\end{array}
$$

The map $q$ is induced by the quotient map from $\prod_{i=1}^{n} X_{i} \rightarrow \bigwedge_{i=1}^{n} X_{i}$ and $D$ denotes the map $\left(\bigvee_{g \in G_{n}} C_{g}\right)$.

We will determine the range in which the side and bottom arrows are injective and/or surjective. From there we will be able to deduce the connectivity of $\Omega T_{n}$.

Let $\Gamma_{g h}^{\prime}: \bigwedge_{i=1}^{n} X_{i} \rightarrow \Omega^{n} \Sigma^{n}\left(\bigwedge_{i=1}^{n} X_{i}\right)$ be the map that takes $x \in \bigwedge_{i=1}^{n} X_{i}$ to the map $\Gamma_{g h} \wedge x$. By Proposition 6.8 and the Freudenthal suspension theorem, $\Gamma_{g h}^{\prime}$ is $2 n(k+1)-2$-connected when $g=h$ and is null homotopic otherwise. The map $\Gamma$ can be represented by the $(n-1) ! \times(n-1)$ ! matrix

$$
\left[\left(\Gamma_{g h}^{\prime}\right)_{*}\right]_{g, h \in G_{n}} .
$$

By the Whitehead theorem and the preceding, it follows that $\Gamma$ is an isomorphism for $m \leq 2 n(k+1)-2$.

By the Künneth theorem, $r$ is an isomorphism for $m \leq 2 n(k+1)-2$, and $q$ is a surjection. Therefore, $\Gamma \circ q$ is a surjection for $m \leq 2 n(k+1)-2$. Since the right arrows are both isomorphisms for $m \leq 2 n(k+1)-2,\left(\Omega T_{n} \circ \bigvee_{g \in G_{n}}\right)_{*}$ is a surjection for $m \leq 2 n(k+1)-2$. In particular, $\Omega T_{n}$ is a surjection for $m \leq 2 n(k+1)-2$.

Since $\pi_{m}\left(\chi_{n}(\Omega \Sigma)\left(X_{1}, X_{2}, \ldots, X_{n}\right)\right)$ and $\pi_{m}\left(\operatorname{Map}_{*}\left(\Sigma \Delta_{n}, X_{1} \wedge X_{2} \wedge \cdots \wedge X_{n}\right)\right)$ are isomorphic to $\pi_{m}\left(\prod_{(n-1) !} \bigwedge_{i=1}^{n} X_{i}\right)$ for $m \leq(n+1)(k+1)-1$, we know that $\widetilde{H}_{m}\left(\chi_{n}(\Omega \Sigma)\left(X_{1}, X_{2}, \ldots, X_{n}\right)\right)$ and $\widetilde{H}_{m}\left(\operatorname{Map}_{*}\left(\Sigma \Delta_{n}, X_{1} \wedge X_{2} \wedge \cdots \wedge X_{n}\right)\right)$ are isomorphic in the same range. Furthermore, the finiteness condition on the spaces $X_{1}, X_{2}, \ldots, X_{n}$ guarantees that these homology groups are finitely generated abelian groups. Hence, the surjection $\Omega T_{n}$ must be an isomorphism for $m \leq(n+1)(k+1)-1$. By the Whitehead theorem, $\Omega T_{n}$ is $(n+1)(k+1)-1$ connected.

\section{REFERENCES}

[B-K] A. K. Bousfield and D. M. Kan, Homotopy limits, completions, and localizations, Lecture Notes in Math., vol. 304, Springer-Verlag, Berlin, 1972.

[B-W] M. G. Barratt and J. H. C. Whitehead, The first non-vanishing group of an $(n+1)$-ad, Proc. London Math. Soc. 6 (3) (1956), 417-439.

[E-S] G. Ellis and R. Steiner, Higher dimensional crossed modules and the homotopy groups of $(n+1)$-ads, J. Pure Appl. Algebra 46 (1987), 117-136.

[G1] T. G. Goodwillie, Calculus I: The first derivative of pseudoisotopy theory, K-Theory 4 (1990), $1-27$.

[G2] _ Calculus II: Analytic functors, K-Theory 5 (1992), 295-332. 
[G3] - Calculus III: The Taylor series of a homotopy functor, K-Theory (to appear).

[Ha] M. Hall, Jr., A basis for free Lie rings and higher commutators in free groups, Proc. Amer. Math. Soc. 1 (1950), 575-581.

[Hi] P. J. Hilton, On the homotopy groups of the union of spheres, J. London Math. Soc. 30 (1955), 154-172.

[Ja] I. M. James, Reduced product spaces, Ann. of Math. 62 (1955), 170-197.

[J] B. Johnson, The derivatives of homotopy theory, Thesis, Brown Univ., 1991.

[M] J. Milnor, On the construction FK, Algebraic Topology: A Student's Guide by J. F. Adams, London Math. Soc. Lecture Note Series 4, Cambridge Univ. Press, Cambridge, 1972, pp. 119-135.

[R] J. Rognes, The rank filtration in algebraic K-theory, Thesis, Princeton Univ., 1990.

[Wa] F. Waldhausen, Algebraic K-theory of topological spaces II, Algebraic Topology-Aarhus 1978, edited by J. F. Dupont and I. H. Madsen, Lecture Notes in Math., vol. 763, SpringerVerlag, Berlin, 1979, pp. 356-394.

[Wh] G. W. Whitehead, Elements of homotopy theory, Springer-Verlag, New York, 1978.

[Wi] E. Witt, Treue Darstellung Liescher Ringe, J. Reine Angew. Math. 177 (1937), 152-160.

Department of Mathematics, Union College, Schenectady, New York 12308

E-mail address: johnsonb@unvax.union.edu 